\title{
Term Structure of Interest Rates with Regime Shifts
}

\author{
Ravi Bansal and Hao Zhou* \\ First Draft: May 1998 \\ This Version: August 2001
}

\begin{abstract}
We develop a term structure model where the short interest rate and the market price of risks are subject to discrete regime shifts. Empirical evidence from Efficient Method of Moments estimation provides considerable support for the regime shifts model. Standard models, which include affine specifications with up to three factors, are sharply rejected in the data. Our diagnostics show that only the regime shifts model can account for the well documented violations of the expectations hypothesis, the observed conditional volatility, and the conditional correlation across yields. We find that regimes are intimately related to business cycles.
\end{abstract}

*We thank Clifford Ball, Tim Bollerslev, Ron Gallant, Pete Kyle, Ken Singleton, George Tauchen, and seminar participants at the Duke Financial Economics Lunch Group, Econometric Society 1999 Summer Meeting, Federal Reserve Board, Michigan State University, and Western Finance Association 2000 Annual Meeting for their helpful suggestions. The views expressed in this paper reflect those of the authors and do not represent those of the Board of Governors of the Federal Reserve System or other members of its staff. We are grateful to an anonymous referee for constructive comments and to the editor Richard Green for his helpful suggestions. For questions and comments, please contact Ravi Bansal, Fuqua School of Business, Duke University, Box 90120, Durham NC 27708 USA, Email ravi.bansal@duke.edu, Phone 919-660-7758, Fax 919-660-8038; or Hao Zhou, Trading Risk Analysis Section, Division of Research and Statistics, Federal Reserve Board, Washington DC 20551 USA, Email hao.zhou@frb.gov, Phone 202-452-3360, Fax 202-7285887. 
Many papers document that the univariate short interest rate process can be reasonably well modeled in the time series as a regime switching process (see Hamilton (1988), Garcia and Perron (1996)). In addition to this statistical evidence, there are economic reasons as well to believe that regime shifts are important to understanding the behavior of the entire yield curve. For example, business cycle expansion and contraction "regimes" potentially have first order effects on inflationary expectations, monetary policy, and nominal interest rates. Such shifts in regimes, on economic grounds, contain the possibility of significant impact not only on the short interest rates but the entire term structure of interest rates. However, standard term structure models, such as the Cox, Ingersoll, and Ross (1985) (CIR) and affine models, do not consider the possibility of discrete changes in regimes despite the potential for sizable effects from such regime shifts. In the context of the U.S. treasury yields, the poor empirical performance of the various versions of the CIR model (see Brown and Dybvig (1986), Gibbons and Ramaswamy (1993), Chen and Scott (1993), and Pearson and Sun (1994)) may well be due to the fact that they do not consider the possibility of discrete regime shifts. ${ }^{1}$

A common approach, as in Gray (1996) and Ang and Bekaert (1998), is to model only the short interest rate as a regime switching process. In this paper we present a model where regime shifts affect the entire term structure of interest rates. Other papers that develop term structure models with regime shifts include Naik and Lee (1997) and Evans (1998). The model presented in this paper differs from that in Naik and Lee (1997) - among other differences, we allow regime shifts to also affect the parameters that govern the market prices of risks. This, as discussed below, is important to capture the conditional second moment properties of the yields and the violations of the expectations hypothesis. Evidence provided in Dai and Singleton (2000b), Ahn, Dittmar, and Gallant (2001), and Jagannathan, Kaplin, and Sun (2000) also indicates that richer dynamics for the market prices of risks may be required to capture the observed yield curve dynamics. In an independent study Evans (1998) studies implications for risk premia on bonds in a model where consumption and inflation are subject to regime shifts. In contrast, the factors and regimes in this paper are treated as latent. Further, our focus is on simultaneously explaining the transition density for nominal yields and the well documented violations of the expectations hypothesis.

To provide a consistent method to estimate various models under consideration we rely

\footnotetext{
${ }^{1}$ In particular, evidence provided by Brown and Dybvig (1986), shows that the parameters of the model change considerably across time; a feature which is consistent with the premise of regime shifts.
} 
on a simulation based estimator for the model. In particular we use the Efficient Method of Moments (EMM), developed in Bansal, Gallant, Hussey, and Tauchen (1995) and Gallant and Tauchen (1996) to estimate all the models under consideration. Tests of over-identifying restrictions based on the EMM method provide a way to compare different, potentially nonnested models. This estimation technique forces the model to confront several important aspects of the data, such as the conditional volatility and correlation across different yields. To provide diagnostics which permit sharp discrimination across models, we rely on the reprojection methods developed by Gallant and Tauchen (1998). The empirical exercise relies on U.S. treasury bills and bonds data from 1964 to 1995.

Our empirical evidence suggests that the benchmark CIR and affine model specifications with up to three factors are sharply rejected with p-values of zero. The only model specification that finds support in the data (with p-value of 14\%) is our preferred two-factor regime switching model where the market price of risks depends on regime shifts. The two-factor regime switching model with restrictions on the market price of risk, related to the Naik and Lee (1997) specification, is also rejected in the data.

The key dimension of discrimination across models lies in their ability to capture the conditional volatility and the conditional cross-correlation across yields. Our preferred regime shifts specification is capable of duplicating these features of the data quite well-affine and CIR specifications have considerable difficulty in matching these aspects of the data. In addition, the preferred regime shifts model specification also can duplicate the violations of the expectations hypothesis as documented in Campbell and Shiller (1991). The affine and CIR specifications have difficulty in matching these violations as well. Exploring the implications for the expectations hypothesis, from the perspective of affine and CIR model specifications is also the focus of Roberds and Whiteman (1999), Backus, Foresi, Mozumdar, and $\mathrm{Wu}$ (2000), and Dai and Singleton (2000a).

Our diagnostics of the various models show that the our preferred regime shifts model specification produces the smallest cross-sectional pricing errors across all the specifications considered in the paper. Further, the extracted regimes are intimately related to the slope of the yield curve and business cycles - regimes with low yield spreads occur prior to or during business contractions (see Harvey (1988), and Estrella and Hardouvelis (1991) for related evidence).

The remainder of this paper is organized in the following manner. Section 1 presents the regime shifts term structure model. Section 2 discusses the empirical evidence-model 
estimation and specifications test, an array of diagnostics based on the conditional correlation and volatility, cross-sectional implications on pricing errors, violations of the expectation hypothesis, and the link between regime classification and business cycles. The technical details on implementing the simulation-based EMM estimator is supplied in Appendix D. The last section presents concluding comments.

\section{Models for the Term Structure of Interest Rates}

In this section, we present the term structure model which incorporates regime shifts. The derivation focuses on a single factor (i.e., state variable) as the extensions to the multifactor case follow immediately from the single square-root factor case and are discussed in Appendix B and C. Our discrete time analysis to characterize the term structure is related to that in Sun (1992), Campbell, Lo, and MacKinlay (1997), and Backus and Zin (1994). It is important to note that the solution to the yield curve in the regime shifts model relies on a log-linear approximation.

To fix ideas it is useful to first consider the benchmark 1-Factor[CIR] model. The key building block of this benchmark case is the state variable $x_{t}$ which follows the square-root process,

$$
x_{t+1}-x_{t}=\kappa\left(\theta-x_{t}\right)+\sigma \sqrt{x_{t}} u_{t+1},
$$

where $u_{t+1} \sim N(0,1)$ is a white noise, $\kappa$ is the mean reversion parameter, $\theta$ is the longrun mean parameter, and $\sigma$ is the local variance parameter. Based on (1), it follows that $E_{t}\left[x_{t+1}\right]=x_{t}+\kappa\left(\theta-x_{t}\right)$, and $\operatorname{Var}_{t}\left(x_{t+1}\right)=\sigma^{2} x_{t}$. This is the discrete time counterpart to the CIR model, discussed in considerable detail in Sun (1992) and Campbell et al. (1997). We further assume that the pricing kernel (in equilibrium, the inter-temporal marginal rate of substitution), $M_{t+1}$, can be written as,

$$
M_{t+1}=\exp \left\{-r_{f, t}-\left(\frac{\lambda}{\sigma}\right)^{2} \frac{x_{t}}{2}-\frac{\lambda}{\sigma} \sqrt{x_{t}} u_{t+1}\right\} .
$$

Note that $\mathrm{E}_{t}\left[M_{t+1}\right]=\exp \left(-r_{f, t}\right)$, where $r_{f, t}$ is the continuous one period risk-free rate-the conditional mean of the pricing kernel must equal the price of the one period default-free discount bond. Sun (1992) also shows that this discrete time specification converges to the continuous-time specification when the interval across adjacent time periods shrinks. The CIR specification, and its multi-factor extensions are special cases of the regime shifts model which we discuss next. 
We are interested in deriving the implications for the term structure when the economy is subject to regime shifts. To keep things tractable we will model the regime shifts process as a two state Markov process as in Hamilton (1989). Suppose that the evolution of tomorrow's regime $s_{t+1}=0,1$ given today's regime $s_{t}=0,1$ is governed by the transitional probability matrix of a Markov chain

$$
\Pi=\left[\begin{array}{ll}
\pi_{00} & \pi_{01} \\
\pi_{10} & \pi_{11}
\end{array}\right],
$$

where $\sum_{j=0,1} \pi_{i j}=1$ and $0<\pi_{i j}<1 .^{2}$ To emphasize the uncertainty of future regime in the following structural development, we use $s_{t+1}$ instead of $j$. In addition to the discrete regime shifts of the economy, as in the benchmark case, the economy is also affected by a continuous state variable,

$$
x_{t+1}-x_{t}=\kappa_{s_{t+1}}\left(\theta_{s_{t+1}}-x_{t}\right)+\sigma_{s_{t+1}} \sqrt{x_{t}} u_{t+1},
$$

where $\kappa_{s_{t+1}}, \theta_{s_{t+1}}$, and $\sigma_{s_{t+1}}$, are the regime-dependent mean reversion, long run mean, and volatility parameters respectively. All these parameters are subject to discrete regime shifts. Specifically, $x_{t+1}-x_{t}=\kappa_{0}\left(\theta_{0}-x_{t}\right)+\sigma_{0} \sqrt{x_{t}} u_{t+1}$ if the regime $s_{t+1}=0$, and $x_{t+1}-x_{t}=$ $\kappa_{1}\left(\theta_{1}-x_{t}\right)+\sigma_{1} \sqrt{x_{t}} u_{t+1}$ if the regime $s_{t+1}=1$. Note that the innovation in the process (4), $u_{t+1}$, is conditionally normal given $x_{t}$ and $s_{t+1} \cdot{ }^{3}$ It is also assumed that the agents in the economy observe the regimes, though the econometrician may possibly not observe the regimes.

The pricing kernel for this economy, is similar to that in the benchmark case, save for incorporating regime shifts

$$
M_{t+1}=\exp \left\{-r_{f, t}-\left(\frac{\lambda_{s_{t+1}}}{\sigma_{s_{t+1}}}\right)^{2} \frac{x_{t}}{2}-\frac{\lambda_{s_{t+1}}}{\sigma_{s_{t+1}}} \sqrt{x_{t}} u_{t+1}\right\}
$$

In the standard representative agent economy with log preferences, the equilibrium pricing kernel (or IMRS) depends on the consumption and inflation process - the above specification of the pricing kernel captures the intuition that these aggregate processes are latent and subject to regime shifts (as in Hamilton (1989)). The details of this general equilibrium

\footnotetext{
${ }^{2}$ When the transition probability is independent of the regime, that is when $\pi_{00}=\pi_{10}$ we get a nonpersistent (iid) regime switching process. Note that this probability restriction implies that $\pi_{01}=1-\pi_{00}=$ $\pi_{11}$, and $\pi_{00}+\pi_{11}=1$. Further $\pi_{00}+\pi_{11}-1$, as shown in Hamilton (1990), determines the persistence in the regime switching process, which in the iid case is zero.

${ }^{3}$ For analytical tractability we assume that the process for regime shifts $s_{t+1}$ is independent of $x_{t+1-l}$, $l=0 \cdots \infty$, this is similar to the assumptions made in Hamilton's regime switching models.
} 
argument which motivates (5) are presented in Appendix A. Note that the $\lambda$ parameter that affects the risk premia on bonds is also subject to regime shifts and hence depends on $s_{t+1}$.

With regime shifts, we conjecture that the bond price with $n$ periods to maturity, at date $t$ depends on the regime $s_{t}=i, i=0,1$, and $x_{t}$

$$
P_{i}(t, n)=\exp \left\{-A_{i}(n)-B_{i}(n) x_{t}\right\}
$$

The one period ahead bond price, analogously depends on $s_{t+1}$ and $x_{t+1}$

$$
P_{s_{t+1}}(t+1, n-1)=\exp \left\{-A_{s_{t+1}}(n-1)-B_{s_{t+1}}(n-1) x_{t+1}\right\} \text {. }
$$

In addition we impose the boundary condition $A_{i}(0)=B_{i}(0)=0$ and the normalization $A_{i}(1)=0, B_{i}(1)=1$, for $i=0,1$, that is, $r_{f, t}=x_{t}$. Setting $r_{f, t}=x_{t}$ and using a log-linear approximation, the key asset pricing condition that follows is (see Appendix A.3 for details)

$$
\sum_{s_{t+1}=0,1} \pi_{i s_{t+1}}\left[-x_{t}+\mu_{n, s_{t+1}, t}+\frac{\sigma_{n, s_{t+1}, t}^{2}}{2}+B_{s_{t+1}}(n-1) \lambda_{s_{t+1}} x_{t}\right]=0
$$

with $i=0,1$. The conditional mean and volatility of the bond return in regime $s_{t+1}$ is $\mu_{n, s_{t+1}, t}$ and $\sigma_{n, s_{t+1}, t}^{2}$ respectively. With discrete regime shifts, (6) is the asset pricing restriction on the risk premium. It is important to note that the risk premium depends only on the current regime $s_{t}$ and $x_{t}$. Specifically note that (6) can equivalently be stated as

$$
E_{t}\left[\mu_{n, s_{t+1}, t}+\frac{\sigma_{n, s_{t+1}, t}^{2}}{2}-r_{f, t} \mid x_{t}, s_{t}\right]=-x_{t} E_{t}\left[B_{s_{t+1}}(n-1) \lambda_{s_{t+1}} \mid s_{t}\right]
$$

Note that all risk-premiums and bond prices at date $t$ depend only on $s_{t}$ (the current regime) and $x_{t}$. To further get some intuition regarding the risk premium result, note that $-\sigma_{s_{t+1}} B_{s_{t+1}}(n-1) \sqrt{x_{t}}$ is the exposure of the bond return to the standardized (i.e., $N(0,1)$ ) shock $u_{t+1}$ in regime $s_{t+1}$. Further, $\left[\frac{\lambda_{s_{t+1}}}{\sigma_{s_{t+1}}} \sqrt{x_{t}}\right]$ is the exposure of the pricing kernel (more precisely, $\left.-M_{t+1} / E_{t}\left[M_{t+1}\right]\right)$ to $u_{t+1}$ in regime $s_{t+1}$. The covariance between these exposures determine the compensation for risk in regime $s_{t+1}$. Hence, the risk compensation for regime $s_{t+1}$ is the product

$$
-\sigma_{s_{t+1}} B_{s_{t+1}}(n-1) \sqrt{x_{t}} \times\left[\frac{\lambda_{s_{t+1}}}{\sigma_{s_{t+1}}} \sqrt{x_{t}}\right]=-B_{s_{t+1}}(n-1) \lambda_{s_{t+1}} x_{t}
$$

Given information regarding $s_{t}$ (the current regime), $x_{t}$, and the regime transition probabilities; agents integrate out the future regime, $s_{t+1}$, which leads to the risk premium result stated in (7). 
The market price of risk, as discussed in Cochrane (2001), can be computed as the risk premium on an asset with a unit exposure to the source of (standardized) risk. Consequently, using the above analysis, an asset return with an exposure of -1 to the standardized shock $u_{t+1}$ would have a risk premium of $-E_{t}\left[\frac{\lambda_{s_{t+1}}}{\sigma_{s_{t+1}}} \mid s_{t}\right] \sqrt{x_{t}}$; this is the risk premium per unit of standard deviation, and hence the market price of risk. Using exactly the same logic, it also follows that the market price of risk in the 1-Factor[CIR] model is, $-\frac{\lambda}{\sigma} \sqrt{x_{t}} .4$

In the regime shifts model the continuous bond returns, from the perspective of economic agents, are distributed as a conditional mixture of normals. In the standard case, bond returns are conditionally normal. The risk premium restriction, (6), also shows that agents anticipate the effects of regime shifts and are compensated for it. For example, if the probability of the high risk regime is greater when $s_{t}=0$, then agents will demand a greater risk premium in regime $s_{t}=0$, relative to when they are in regime $s_{t}=1$. Further, if regimes are fairly persistent, then the effects of a regime switch on the risk premium and hence the term structure will be infrequent, but potentially large when a regime switch does happen. This feature, in addition to the implied distribution for bond returns (and yields) distinguishes the regime switching model from the standard 1-Factor[CIR] model discussed above.

From (6) it is evident that the risk premium on bonds depends on the current regime $i, x_{t}$ and is also governed by the regime-dependent risk parameters $\lambda_{0}$ and $\lambda_{1}$. If the risk parameters across two regimes are restricted to be the same (i.e., $\lambda_{0}=\lambda_{1}$ ), we arrive at a specification related to that in Naik and Lee (1997). Allowing the risk parameters to depend on the regimes opens an additional channel for regime shifts to affect bond prices. This feature, as discussed below, is important for understanding many of the quantitative features of term structure data.

Given (6), the solution for the bond prices can be derived, by solving for the unknown coefficients $A$ and $B$, in particular we show;

$$
\left[\begin{array}{l}
B_{0}(n) \\
B_{1}(n)
\end{array}\right]=\left[\begin{array}{ll}
\pi_{00} & \pi_{01} \\
\pi_{10} & \pi_{11}
\end{array}\right]\left[\begin{array}{l}
\left(1-\kappa_{0}-\lambda_{0}\right) B_{0}(n-1)-\frac{1}{2} \sigma_{0}^{2} B_{0}^{2}(n-1)+1 \\
\left(1-\kappa_{1}-\lambda_{1}\right) B_{1}(n-1)-\frac{1}{2} \sigma_{1}^{2} B_{1}^{2}(n-1)+1
\end{array}\right]
$$

and

$$
\left[\begin{array}{l}
A_{0}(n) \\
A_{1}(n)
\end{array}\right]=\left[\begin{array}{ll}
\pi_{00} & \pi_{01} \\
\pi_{10} & \pi_{11}
\end{array}\right]\left[\begin{array}{l}
A_{0}(n-1)+\kappa_{0} \theta_{0} B_{0}(n-1) \\
A_{1}(n-1)+\kappa_{1} \theta_{1} B_{1}(n-1)
\end{array}\right]
$$

\footnotetext{
${ }^{4}$ Note that the market prices of risks for additional square-root factors, $x_{t+1}$, can be characterized in an analogous manner.
} 
with initial conditions $A_{0}(0)=A_{1}(0)=B_{0}(0)=B_{1}(0)=0$. As these coefficients depend on the current regime - the current yield curve consequently depends only on the current regime and $x_{t}$. Note that bond price coefficients are mutually dependent on both the regimescurrent bond prices reflect agent's expectations regarding regime shifts in the future.

Finally, the bond yield of a $K$ factor regime-switching model can be derived in an analogous manner (see Appendix C for details),

$$
Y_{s}(t, n)=-\frac{\ln P_{s}(t, n)}{n}=\frac{A_{s}(n)}{n}+\sum_{k=1}^{K} \frac{B_{k s}(n) x_{k t}}{n} .
$$

It is worth noting that the yield curve restrictions, such as (10), can be derived from a representative agent based general equilibrium model of the type considered in Lucas (1978) and is briefly discussed in Appendix A. Independently, Evans (1998), also presents a related general equilibrium model which incorporates regime shifts.

\section{Estimation Results and Model Diagnostics}

\subsection{Methodology}

In estimation our focus will be to evaluate if the different models can justify the observed behavior of two interest rates - the six month U.S. t-bill rate, and the five year U.S. tbond rate. We explore the ability of benchmark CIR and affine models with up to a three latent factors, and two factor regime switching models to justify the observed conditional distribution of the two interest rates under consideration. To utilize a consistent approach for evaluation and estimation across the different models we rely on simulation based EMM (efficient method of moments) estimator, developed in Bansal et al. (1995) and Gallant and Tauchen (1996).

The EMM estimator consists of two steps. First, the empirical conditional density of observed interest rates is estimated by a semi-nonparametric (SNP) series expansion. This SNP expansion has a VAR-ARCH Gaussian density as its leading term, and departures from the Gaussian leading term are captured by a Hermite polynomial expansion. Second, the score functions from the log-likelihood of the SNP density are used as moments to construct a GMM type criterion function. The scores are evaluated using the simulation output from a given term structure model and the criterion function is minimized with respect to the parameters on the term structure model under consideration. By using the 
scores from the non-parametric SNP density as the moment conditions, the model is forced to match the conditional distribution of the observed 6-month and 5-year yields. This is a GMM-type estimator which in addition to providing comparable (across models) measures for specification tests, also permits a series of interesting diagnostics to understand the advantages and shortcomings of the different models under consideration. In particular, the normalized objective function acts as an omnibus specification test, which is distributed as a chi-square (as in GMM) with degrees of freedom equal to the number of scores (moment conditions) less the number of parameters in the particular term structure model.

Note that the distance matrix (the weight matrix in GMM) used in constructing the specification test is identical across different model specifications (the null hypotheses). Consequently, the p-values based on this specification test can be directly compared across different structural models to identify the best model specification. ${ }^{5}$ It is well recognized in the literature, that tests for the absence of regime-shifts against a regime shifting alternative require non-standard approaches (see Hansen (1992) and Garcia (1992)). Our approach of comparing all the considered models to a common non-parametric density (the SNP density), allows us to rank order all the considered models according to the p-values implied by the EMM criterion function. The advantage of using the non-parametric SNP, as discussed in Gallant and Tauchen (1999), is that it can asymptotically converge to virtually any smooth distributions, including mixture distributions (as is the case with a model of regime shifts). ${ }^{6}$ Greater details regarding the non-parametric SNP and the EMM estimator are provided in the Appendix D.

\subsection{Data Description}

The data set ranging from June 1964 to December 1995, is obtained from the Center for Research in Security Prices (CRSP). There are total 379 monthly observations, with nine maturities 1, 3, 6, 9 month and 1, 2, 3, 4, 5 year. Longstaff and Schwartz (1992), also use this data set to estimate their term structure model. It is important to recognize that the data period 1964-1995 contains five major recessions and six major expansions, which as

\footnotetext{
${ }^{5}$ For a discussion of the importance of having the same distance matrix, for a consistent comparison across models, see Hansen and Jagannathan (1997).

${ }^{6}$ Further, Gallant and Tauchen (1999) show that EMM can provide more efficient estimates relative to alternative method of moment estimators such as simulated method of moments (see Duffie and Singleton (1993)). As is typical with GMM-type estimators, recent Monte Carlo studies have documented the overrejection bias of EMM (see Andersen, Chung, and Sørenson (1999) and Zhou (2001)), in small to moderate sample sizes, therefore the test is more conservative and cautious in model selection.
} 
stated earlier provides potential economic motivation for incorporating regime shifts. In the latter part of the paper we will show an intimate link between business cycles and extracted regimes based on the estimated term structure model.

**********************Insert Table 1 Here $* * * * * * * * * * * * * * * * * * * *$

The summary statistics of these monthly yields are given in Table 1. It is clear that on average, the yield curve is upward sloping. The standard deviation, positive skewness, and kurtosis are systematically higher for short maturities than for long ones. Many of the important dynamics of the two yields are recovered by the estimated SNP density.

To incorporate important time-series and cross-sectional aspects of term structure data we focus on a short term and a long term yield - the yield on the six month bill and the five year note. All the structural models, one, two, three factor with or without regime-switching are forced to match the conditional bivariate joint dynamics of these two yields. We did not use the one month or three month yield to represent the short end, because they are more likely to be influenced by liquidity needs. ${ }^{7}$ The time series plots of the basis yields are reported in Figure 1.

$* * * * * * * * * * * * * * * * * * * * \operatorname{Insert~} \mathrm{Figure} 1 \mathrm{Here} * * * * * * * * * * * * * * * * * * * *$

The estimation results of the chosen non-parametric SNP specification are reported in Table 2 and 3 (see Table A1 in Appendix D for technical notations of the SNP specifications). In the first step of EMM procedure, we tried to fit a seminonparametric (SNP) density of the bivariate joint dynamics of the two basis yields. This SNP density is a Hermite polynomial expansion of a normal density with the leading term as VAR-in-mean and ARCH-in-standard deviation.

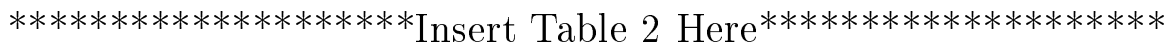

$* * * * * * * * * * * * * * * * * * * *$ Insert Table 3 Here $* * * * * * * * * * * * * * * * * * * *$

Table 2 reports the choice of SNP density and the BIC (Bayesian information criterion) value, based on which we choose our preferred specification. The leading term of the bivariate SNP density, being conditionally normal, has 1 lag in the VAR based conditional

\footnotetext{
${ }^{7}$ See Bansal and Coleman (1996) for such a macroeconomic model and Liu, Longstaff, and Mandell (2000) for term structure evidence.
} 
mean (notationally $L_{\mu}=1$ ) and 5 lags in ARCH specification $\left(L_{r}=5\right.$ ). If all other terms in the SNP density were zeroed out, then the SNP density specification would indeed be this conditional normal density. However, the preferred specification allows for departures from conditional normality, in particular, it chooses a polynomial of order $4\left(K_{z}=4\right)$ in the standardized residual $z$; this overall specification of the non-parametric density leads to a "semiparametric ARCH" specification for the bivariate data. This specification is similar to that proposed by Engle and González-Rivera (1991). This specification allows for skewness and kurtosis in the SNP distribution. The total number of parameters for this semi-parametric ARCH specification is 28 , that is $l_{\theta}=28$. Hence the total number of scores that we have from this SNP density is also 28. Note that these scores serve the role of moment restrictions in a method of moments type estimation. The information criterion for choosing the preferred SNP specification is the minimum value of BIC provided in Table 2. Table 3 gives the parameter estimates of the SNP density. The preferred specification, for future reference is, $L_{\mu}=1, L_{r}=5, K_{z}=4$. Figure 2 presents a plot of the bivariate density. ${ }^{8}$

$* * * * * * * * * * * * * * * * * * * * \operatorname{Insert}$ Figure 2 Here $* * * * * * * * * * * * * * * * * * * *$

Given the estimated non-parametric SNP density it is possible to compute the conditional moments of the observed interest rates analytically. It is fairly instructive to focus on some specific aspects of the estimated non-parametric SNP bivariate density. The top panels in Figure 7 and Figure 8 give the estimated conditional volatilities of the 6-month and 5-year yields, which seem to be very persistent and fairly volatile. The short interest rate has a wide range for the conditional volatility which peaks around 1980 . The range for the five year yield volatility is narrow, relative to that of the 6-month yield. The top panel of Figure 9 provides information regarding the conditional covariance across the two yieldsnote that this covariance varies considerably, and also peaks in the early 80's. The top panel in Figure 10 shows the conditional correlation between the 6-month and 5-year yields, the range for this correlation is from about $40 \%$ to $90 \%$ - a wide range indeed. The most volatile period for bond yields, the early 80's sees, is associated with a considerable drop

\footnotetext{
${ }^{8}$ Note that in our preferred specification the interaction terms above degree 2 are suppressed (i.e, $I_{z}=2$ ), and the coefficients of the polynomial do not depend on the lagged values of the interest rates- $x$, that is $K_{x}=0$. When the $K_{x}=0$, it also follows that the $x$-polynomial as coefficients of $z$-polynomial are all constants, hence these coefficients are not depending on lag state $x$, and in this case $L_{p}=1$. For greater details see Appendix D, Gallant and Tauchen (1989), and Tauchen (1997).
} 
in the conditional correlation. The behavior of the conditional variance and the crosscorrelation, as documented above, poses a serious challenge to the various term structure models under consideration. Moreover, these features of the data are critical for pricing interest rate sensitive derivative securities. Hence, these dimensions of the data are important for evaluating the plausibility of term structure models. Jagannathan et al. (2000) also highlight the economic importance of matching the conditional volatility or interest rates, and the difficulties that benchmark specifications, such as the CIR model in matching the volatility of interest rates.

\section{$2.3 \quad$ Estimation Results and Goodness-of-Fit}

Table 4 gives the main EMM estimation results for different models: one-factor squareroot (1-Factor[CIR]), one-factor regime-switching (1-Factor[RS]), two-factor square-root (2Factor[CIR]), two-factor regime-switching (2-Factor[RS]), two-factor regime-switching model with restrictions on the risk premium parameters (that is $\lambda_{10}=\lambda_{11}$ and $\lambda_{20}=\lambda_{21}$ ) related to the Naik and Lee (1997) specification (2-Factor[RSc]), three-factor square-root (3Factor[CIR]), and three-factor affine (3-Factor[AF]). The results reported here are for simulation size of 50,000. The square-root factors are generated monthly, and the bond price formula given in (10) is applied with one month being the time interval - consequently, the five year bond is treated as a 60 month bond (similarly for the 6-month bond). The data that was used for estimation was the monthly yield multiplied by 12, analogously the simulated monthly yields are also multiplied by 12 . Note that the optimized objective function of EMM estimation serves as a specification test, which is distributed as a chi-square with degree of freedom equals to 28 (the number of SNP scores) minus the number of structural parameters (varying across different models).

*********************** Insert Table 4 Here $* * * * * * * * * * * * * * * * * * * * *$

The single factor CIR model (1-Factor[CIR]) is sharply rejected with p-value less than 0.0000. The magnitudes and signs of the parameter estimates are close to those reported in the literature for the monthly frequency. The one factor model with regime-shifts (1Factor[RS]) is also rejected with a p-value less than 0.0000. It is worth noting that the regimes do not differ much in the mean reversion coefficient or the long run mean. The key difference is in the volatility; regime 0 volatility $\sigma_{0}$, is more than twice that in the low volatility regime, regime 1 . In addition, the parameter that governs the risk premium, $\lambda$, 
is larger in the low volatility regime. All the parameters are estimated with small standard errors.

The two factor square-root model (2-Factor[CIR]) improves things but this specification is still sharply rejected - the model specification test drops to 62.376 with p-value smaller than 0.0000. Since the factors additively determine the short rate, the long run mean levels are about half of the one factor model. The first factor has higher mean reversion and a larger variance parameter, relative to the second factor. All parameter estimates are significant, except the risk premium parameter for the first factor, which is not significantly different from zero. The inclusion of a second factor provides a significant improvement over the one factor benchmark model, though relative to the regime shifts one factor model, the improvement at best is marginal.

Next consider the 2-Factor[RSc] specification in which the risk parameters are constrained to be the same across regimes. This specification does provide an improvement over the standard 2-Factor[CIR] model, however the test statistic has a value of 45.067 and the specification is still rejected with a p-value of 0.0000 . The regime probabilities are very persistent (0.95 and 0.92). Some parameters are estimated with relatively large standard errors $\left(\lambda_{10}, \kappa_{11}, \theta_{21}\right.$, and $\left.\kappa_{21}\right)$. The long run mean, mean reversion and local variance parameters are not significantly different across regimes for the first factor, but are quite different across regimes for the second factor.

The best model amongst all specifications is the two-factor regime switching specification which allows the risk premium parameters (i.e., the $\lambda$ 's) to also depend on the regimes. This specification finds considerable support in the data, as the p-value for this specification is about $15 \%$, which is well above conventional levels of significance. In terms of the parameters of interest, note that the first factor, for both regimes (i.e., 0 and 1), relative to the second factor has far greater mean reversion. For both regimes, the first factor is also more volatile relative to the second factor. The regime 1 volatility parameter is larger for both the factors, and in this sense it represents the more volatile regime. Additionally, the risk premium parameter, for both factors, is larger for regime 1, the more volatile regime. The regime probabilities (standard error) are $\pi_{11}=0.91(0.22)$ and $\pi_{00}=0.94(0.18)$. All the parameters of the model are estimated fairly accurately. These transition probabilities reported for the 2-Factor[RS] specification, are comparable to those found in other papers (see Gray (1996), Hamilton (1988), and Cai (1994)). 
The 2-Factor[RS] model (and the 2-Factor[RSc] model as well) can be viewed as a 3 factor model with the regime switching factor being a multiplicative or nonlinear switching process. For a fair comparison of this two factor regime switching model, we also estimate a three factor CIR model. As Table 4 shows, the 3-Factor[CIR] model is also sharply rejected with chi-square being 45.607 and p-value of 0.0001. All the parameter estimates are significant, and all factors are associated with a positive risk premium. The first factor has a high long run mean, relatively high mean reversion of 0.033 , and a relatively smaller variance. The other two factors have greater persistence and volatility. Our empirical evidence suggests that the standard three factor CIR model cannot account for the observed behavior of the short and long yields used in this empirical exercise.

Using the estimated parameter values and its estimated variance-covariance matrix in our preferred 2-Factor[RS] model, one can perform the $t$-test for some interesting hypotheses. These test statistics suggest that the regime shifts are not an iid mixture $\left(t_{\pi_{00}=1-\pi_{11}}=\right.$ 2.1507). The first factor has significant regime switches in level, mean reversion, and volatility parameters - the t-ratios are $t_{\theta_{10}=\theta_{11}}=7.4972, t_{\kappa_{10}=\kappa_{11}}=2.7699, t_{\sigma_{10}=\sigma_{11}}=45.9025$, respectively. Note that the risk parameters are significantly different across regimes, $t_{\lambda_{10}=\lambda_{11}}=$ 35.1633. This is indicative of the reasons for the rejection of the 2-Factor[RSc] specification, which forces $\lambda_{10}$ to equal $\lambda_{11}$. Evidence for significant differences in regimes in the second factor is muted, with the t-ratios for the level, mean reversion, volatility, and risk parameters, being $t_{\theta_{20}=\theta_{21}}=1.7521, t_{\kappa_{20}=\kappa_{21}}=5.6031, t_{\sigma_{20}=\sigma_{21}}=3.4267, t_{\lambda_{20}=\lambda_{21}}=0.3763$, respectively.

A recent study by Dai and Singleton $(2000 \mathrm{~b})$ found that a three-factor affine term structure model, similar to that proposed by Chen (1996), passes the standard statistical test of over-identifying restrictions. The Dai and Singleton (2000b) paper uses swap yields data after 1987 for their empirical exercise. By confining their study to the swap interest rate data beginning in 1987, much of the information regarding the behavior of interest rates in recessions, e.g., 1979-1982, and in 1973-76, does not bear on the estimation. The sample period from 1964 to 1995, has economically important features that are missing from the post 1987 period. For example, there is only one short lived recession in early 90's in the post 1987 sample period. To exhaust alternative affine specifications, we also present empirical results for a three factor affine (labeled here as 3-Factor[AF]). More specifically we focus on a specification, that in Dai and Singleton (2000b) is labeled as $\operatorname{ATS} M_{1}(3)$ - they find considerable empirical support for this specification using the post 1987 swap yield data. 
The discrete time counterpart to this affine specification is;

$$
\begin{aligned}
& x_{1 t+1}-x_{1 t}=\kappa_{1}\left(\theta_{1}-x_{1 t}\right)+\sigma_{1} \sqrt{x_{1 t}} u_{1 t+1} \\
& x_{2 t+1}-x_{2 t}=\kappa_{2}\left(\theta_{2}-x_{2 t}\right)+\sigma_{2} u_{2 t+1}+\sigma_{23} \sqrt{x_{1 t}} u_{3 t+1} \\
& x_{3 t+1}-x_{3 t}=\kappa_{3}\left(x_{2 t}-x_{3 t}\right)+\sqrt{x_{1 t}} u_{3 t+1}+\sigma_{31} \sigma_{1} \sqrt{x_{1 t}} u_{1 t+1}+\sigma_{32} \sigma_{2} u_{2 t+1}
\end{aligned}
$$

Associated with this 3-Factor[AF] specification are three market price of risk parameters, which as before we label as $\lambda_{k}, k=1,2,3$. In all there are 13 parameters to estimate. As reported in Table 4 the 3 -Factor[AF] specification is sharply rejected with a $\mathcal{X}^{2}(15)=76.489$ and a p-value of 0.0000. Our diagnostics, discussed in greater detail below, suggest that the affine models can to a certain extent capture the conditional correlation of yields, however it fall short of matching the conditional volatilities and conditional covariances. In all, our specification tests provide considerable support for the two factor, regime switching model. The considered CIR and affine specifications seem to have considerable empirical difficulties in capturing the nominal yield dynamics.

\subsection{Diagnostics for Different Model Specifications}

In the rest of this section we will provide a range of diagnostics which allow us to understand the strengths and weaknesses of the different model specifications. In particular, these diagnostics reveal why the 2-Factor[RS] specification finds considerable empirical support, while the other specifications discussed above, do not. The diagnostics include looking at the t-ratios for the fitted scores, the re-projected density with its implications for the conditional volatility and cross-correlation for observed yields, and a comparison of the model implied term structure relative to that seen in the data. Here we first look at the t-ratios for the fitted scores, which are directly linked to the EMM estimation procedure.

******************** Insert Table 5 Here $* * * * * * * * * * * * * * * * * * * *$

Table 5 reports the t-tests for the 28 moment conditions for the various models. These 28 scores (moment conditions), for a reasonable model specification, should be close to zero. The size of the t-ratios gives a sense of the scores which different model specifications have difficulty in fitting.

For reference note that for the Hermite polynomial part, $a(i, j)$ refers to the parameter before the polynomial term with $i$-th degree of power on the first variable (6 month yield) and $j$-th degree of power on the second variable ( 5 year yield). For the VAR mean part, 
$\mu(i, j)$ represents the parameter in the $i$ th equation $(i=1$ for the short rate and $i=2$ for the long rate) of the $j$ 'th variable $(j=0$ for the constant, $j=1$ for the lag one short rate, and $j=2$ for the lag one long rate). For the $\mathrm{ARCH}$ standard deviation part, $R(i, j)$ denotes the parameter on variable $i$ with $j$ lags, and the last term $R(3)$ is simply the constant covariance parameter. If the structural model under consideration matches the particular moment under consideration, then at conventional $5 \%$ level of significance the t-ratio should be smaller than 1.96 .

In the context of the benchmark model, it is clear that the three factor (see column 3Factor[CIR]) model provides considerable improvement over the one (1-Factor[CIR]) and two factor (2-Factor[CIR]) specifications. The t-ratios for almost all scores are relatively smaller for the three factor specification. However, in an absolute sense the 3-Factor[CIR] and the 3Factor[AF] models have considerable difficulty in matching many of the Hermite polynomial scores and the ARCH specification scores. This suggests that these benchmark three factor models cannot capture the conditional skewness, kurtosis, and the second moment properties of the bivariate interest rate process. Further, relative to the 3-Factor[CIR] model, the 3-Factor[AF] has considerable difficulties in matching the $\mathrm{ARCH}$ scores - in this sense the 3 -Factor[CIR] specification looks better. Incorporating regime shifts seem to help match the scores considerably better. The regime shifts specification where the parameters that govern the risk premia are restricted across regimes (2-Factor[RSc]) seems to miss both the nonGaussian (the Hermite part) and the ARCH scores. The preferred model, the 2-Factor[RS] specification does quite well in matching all the moments save one in the Hermite polynomial and four in the conditional standard deviation. The magnitude of the highest t-ratio is 3.13 (see ARCH, $R(2,5)$ ), which relative to the size of the t-ratios for other specifications is quite small. Overall, this preferred specification also seems to have the greatest difficulty in matching the conditional volatility and covariance, i.e., the ARCH scores-though, it provides considerable improvement over all other models. The 2-Factor[RS] specification provides large gains in fitting the non-Gaussian polynomials (i.e., the Hermite polynomial parameters), suggesting that conditional skewness and kurtosis in the standardized innovations can be accounted for by the preferred 2-Factor[RS] term structure model.

\subsection{Pricing Error, Implied Regime, and Business Cycle}

In this section we document the cross-sectional differences across models - that is, the ability of different specifications to reproduce the observed yield curve at each date in the data. To 
avoid clutter we focus only on the empirically plausible specifications - the 2-Factor[CIR], the 2-Factor[RSc], 3-Factor[CIR], 3-Factor[AF], and the preferred 2-Factor[RS] specification.

To derive the model implied term-structure for each date, in the context of the two or three factor CIR and affine models, we simply recover the latent factors, by using the bond pricing function, and the estimated parameters,

$$
\mathbf{X}_{\mathbf{t}}=\mathbf{B}^{-1}\left[\mathbf{Y}_{\mathbf{t}}-\mathbf{A}\right] .
$$

The $K$ vector of latent factors $\mathbf{X}_{\mathbf{t}}$, can be recovered by using $K$ observed basis yields, the estimated $K \times K$ matrix of bond pricing parameters $\mathbf{B}$, and the vector $\mathbf{A}$. Given the vector $\mathbf{X}_{\mathbf{t}}$, and the estimated parameters, the entire yield curve can be computed for each date in the data. By construction, the computed yield curve will pass through the basis yields. This approach to extract the latent factors is similar to that used in Chen and Scott (1993), and Duffie and Singleton (1997), amongst others. Note that in the 2-Factor[CIR] model, the computed yield curve will pass through two basis yields and in the 3-Factor[CIR] or 3 -Factor[AF] model through three basis yields.

To compare models, for each date we also compute the absolute average cross-sectional pricing error, with $\hat{Y}(n)_{t}$ being the computed yield of maturity $n$ at date $t$ for a given model specification,

$$
P E_{t}=\frac{\sum_{n=1}^{N}\left|\hat{Y}(n)_{t}-Y(n)_{t}\right|}{N} .
$$

Smaller the pricing error, better is the model specification. The pricing errors will arise due to model misspecification. ${ }^{9}$

To compute the yield curve for each date, under the null specification of 2-Factor[RSc] or 2-Factor[RS] model, one also needs to know the regime at each date. Recall, the yield curve, in addition to the two latent factors, also depends on the latent (i.e., to the econometrician) regime. To deal with this, we first extract the two latent factors (i.e., $K=2$ ) using (12), the estimated $\mathbf{B}_{\mathbf{s}_{\mathbf{t}}}$ and $\mathbf{A}_{\mathbf{s t}_{\mathbf{t}}}$, for $s_{t}=0$ and $s_{t}=1$. Given the latent factors and the estimated parameters, we compute two potential yield curves for each date, one for $s_{t}=0$ and another for $s_{t}=1$. For each regime, it is straight-forward to compute the pricing error $P E_{t}$, for $s_{t}=0,1$. However, under the null of the 2-Factor[RSc] or 2-Factor[RS] model specification, the pricing error (i.e., $P E_{t}$ ) associated with the regime that truly prevails in the economy at date $t$ will be close to zero. ${ }^{10}$ Hence, the regime that prevails at date $t$ is simply the one

\footnotetext{
${ }^{9} \mathrm{An}$ additional source could be measurement errors in the data.

${ }^{10}$ Note that this procedure under the null of the model, will detect the regime correctly. For example, in
} 
which has the smallest pricing error across regimes. With the knowledge of the regime at date $t$, the model implied yield curve and the pricing error also become available. Note that the computed yield curves and the pricing errors for 3-Factor[CIR] or 3-Factor[AF] and the 2-Factor[RSc] or 2-Factor[RS] can be fairly compared - the three factor specification uses three basis yields, whereas the two factor regime shifts specification relies on two basis yields and an identification procedure for the regime classification.

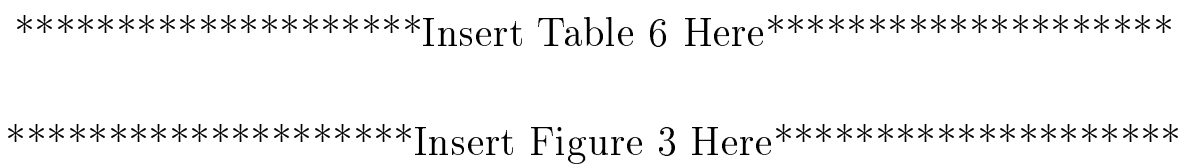

Table 6 provides information regarding the average pricing error (i.e., $\left.\left(\sum_{t}^{T} P E_{t}\right) / T\right)$. It is clear from the sample statistics that the 2-Factor[RS] model has the smallest average pricing error and also the smallest standard deviation in the pricing error. The maximal pricing error associated with the 2-Factor[RS] classification is also the smallest. Further, on average the pricing error is only about 23 basis points for the annualized percentage yields - a small number indeed. The 3-Factor[CIR] and the 3-Factor[AF] specifications have average pricing errors of 25 basis points, which in an absolute sense is also quite small. The 2-Factor[RSc] model has a larger pricing error than the 2-Factor[CIR] and 3-Factor[CIR] model, possibly due to the error of recovering regimes in a rejected model specification. Figure 3 provides the computed yield curves for dates which are 30 months apart - these graphs re-enforce the view that the 2-Factor[RS] specification is the preferred one. The evidence from our specification tests and the reprojection-based diagnostics show that the 2-Factor[RS] model is by far the preferred choice - this view is further corroborated by the cross-sectional evidence.

$* * * * * * * * * * * * * * * * * * * *$ Insert Figure 4 Here $* * * * * * * * * * * * * * * * * * * *$

Figure 4 provides the results from the regime classification. Most of the time, it seems that the economy is in regime 1 . The total number of regime switches recovered from the sample period is 44 , which is larger enough for identifying the regime probabilities. The regime classification by and large coincides with the NBER business cycles. It seems that regime 0 obtains during or around recessions in the economy. The relatively frequent regime shifts between 1991-1995, as shown in Figure 4, reflect the almost identical average pricing our simulations of the 2-Factor[RSc] or 2-Factor[RS] model, this procedure would indeed identify the correct regime - with the pricing error for the true regime being zero. 
error across regimes at those dates. Virtually identical pricing error across regimes for a given date implies that the data is not particularly informative regarding the regime classification.

$* * * * * * * * * * * * * * * * * * * * \operatorname{Insert~Figure~} 5$ Here $* * * * * * * * * * * * * * * * * * * *$

The estimation results discussed earlier suggested that the first factor has considerable differences, especially in volatility, across regimes. Figure 5 shows that the recovered first factor tracks the short yield very well, while the second tracks the long yield. The correlation between the NBER business cycle (recession is regime 0 and boom is regime 1) indicator and the recovered regime indicator is 0.1523 when the early 90 's, where regime identification is poor, is excluded (the analogous correlation for the entire sample is 0.0762). Correlation between NBER business cycle indicator and the yield spread (5 year yield minus 6 month yield) is 0.2342 . Correlation between the model based regime indicator and the yield spread is 0.2299 -that is, regime 1 has high yield spread and regime 0 has low yield spread.

$* * * * * * * * * * * * * * * * * * * *$ Insert Table 7 Here $* * * * * * * * * * * * * * * * * * * *$

Table 7 presents some statistics for the 6-month and 5-year yields across the NBER business cycle regimes (upper panel) and the model-implied regimes (lower panel). It is evident that an important dimension along which economic expansion and contraction periods differ is in terms of the yield spread (for related evidence see Harvey (1988), and Estrella and Hardouvelis (1991)). During NBER expansions the yield spread is 86 basis points while in contractions it is -8 basis points. Our model implied regimes differ across the same dimension - model-implied regime 1 is associated with high yield spreads (83 basis points) and model-implied regime 0 with a yield spread of only -3 basis points. Further, there are similarities between the model based regimes and the NBER business cycle regimes in the behavior of convexity of the yield curve, ${ }^{11}$ and the change in the interest rates (see Table 7). This evidence, in conjunction with the correlation of the model-implied regimes and the NBER business cycles, suggests that our model implied regimes are business cycle regimes. It seems that the model-implied regime 1 is an economic expansion regime and model-implied regime 0 an economic contraction regime. In general, considerable caution should be exercised in interpreting the regime classification as there is considerable error

\footnotetext{
${ }^{11}$ The convexity is computed as $\left[\left(Y_{t}^{3 y r}-Y_{t}^{2 y r}\right)-\left(Y_{t}^{2 y r}-Y_{t}^{1 y r}\right)\right]$, which is a discrete approximation to the second derivative or convexity taking the time interval to be one.
} 
in this classification due to parameter estimation error and model misspecification. ${ }^{12}$ Despite these sources of error, our model-implied regimes have a recognizable association with the business cycle, even though our estimated model is not directly exposed to the NBER recession-expansion indicator.

\subsection{The Expectation Hypothesis Puzzle}

An additional diagnostic is to evaluate if the different model specifications can justify the observed patterns of violations of the Expectations Hypothesis as documented in Campbell and Shiller (1991). Using simulated data from different model specifications, we run the regressions considered in Campbell and Shiller (1991). The difference between the future yield and the current long yield, that is $Y(t+m, n-m)-Y(t, n)$, is regressed on to the normalized yield spread variable, $s_{t}^{(n, m)} \equiv \frac{m}{n-m}[Y(t, n)-Y(t, m)]$, where $n$ is a positive integer of $m$. That is,

$$
Y(t+m, n-m)-Y(t, n)=c_{0}(n, m)+c_{1}(n, m) s_{t}^{(n, m)}+u(n, m)_{t+1} .
$$

In Table 8 we report the estimates for the slope coefficient, $c_{1}(n, m)$, across different $n$ and $m$ in the data and its counterpart for the various models considered in the paper. The "short horizon" results relate to , $n=6,9,12$ months and $m=3,6,9$ months. The "long horizon" results relate to $n=4,5,10$ years and $m=1,2,3$ years. As in Roberds and Whiteman (1999) the reported slope coefficients for the various term structure models are based on 10,000 Monte Carlo replications with a sample size of 379 - the size in the actual data.

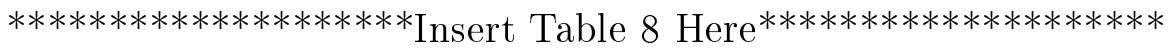

A rise in the long rate maturity $n$ makes the slope more negative in the data, similar to the finding in Campbell and Shiller (1991) and Roberds and Whiteman (1999). ${ }^{13}$ In the short horizon (left panel in Table 8), we find that only the preferred 2-Factor[RS] model can duplicate the violations of the expectations hypothesis documented in the data. All other model specifications fail to duplicate the violations of the expectations hypothesis at the short horizon. Note that the regime shift specification with constant market price of

\footnotetext{
${ }^{12}$ The fact that there is positive pricing error for all dates in both regimes suggests that the regime switching model is misspecified, from the cross-sectional perspective.

${ }^{13}$ For issues regarding small sample estimation bias in the context of testing expectations hypothesis see Bekaert, Hodrick, and Marshall (1997).
} 
risk across regimes (i.e., the 2-Factor[RSc] model) also cannot duplicate the violations of the expectations hypothesis - this underscores the importance, as in the 2-Factor[RS] model, of allowing the market price of risk to depend on regimes. In the long horizon (right panel in Table 8), there seems to be a wedge between the single factor and the multifactor models. We find that the 2-Factor[CIR], the 3-Factor[CIR], 3-Factor[AF], the 2-Factor[RSc], and the 2Factor[RS] models can mimic the violations of the expectations hypothesis to various degrees, but the 1-Factor[CIR] and the 1-Factor[RS] can not. It seems that in the long horizon, the expectations hypothesis regression by itself is not particularly helpful in distinguishing across the different candidate models.

The expectations hypothesis issue is also explored in Roberds and Whiteman (1999). Further, Dai and Singleton (2000a) show that modified affine specifications can also duplicate the violations of the expectations hypothesis. ${ }^{14}$ In addition, Dai (2000) argues that an equilibrium model with habit formation preferences can potentially capture the violations of the expectations hypothesis and the counter-cyclical behavior of risk premia (as in Fama and French (1989)).

\subsection{Reprojected Density and Conditional Second Moments}

The idea behind the reprojection technique (Gallant and Tauchen 1998), is to characterize the dynamics of a given vector of observed variables (i.e., observed yields) conditional on its lags. In models where there are latent factors or regimes, the reprojected density provides a way to characterize the conditional density strictly in terms of observables (i.e., the observed yields). The reprojected density can be estimated by relying on simulated data for the yield series from a given estimated structural model (the null model). In our context, the reprojected density is the bivariate conditional density for the two yields under consideration, the 6-month and the 5-year yield.

Given the estimated null model and the simulated output for yields, the reprojected conditional density is estimated by re-estimating the parameters of the SNP density, using the same specification as was used to characterize the observed bivariate density of the 6-month and 5-year yields earlier (referred to as the unrestricted SNP density). Indeed, any reasonably good term structure model would imply a reprojected conditional density which is very similar to the unrestricted conditional bivariate density for the 6-month and

\footnotetext{
${ }^{14}$ They document that incorporating flexibility in the market price of risks as in Duffee (2000) and Duarte (1999), helps in justifying the violations of the expectations hypothesis.
} 
5-year yields. Once the reprojected density is estimated, specific moments, such as the conditional variances and correlations implied by the model specification can be computed. These conditional moments are simply continuous functions of the conditioning information (i.e., lagged 6-month and 5-year yields) used to estimate the re-projected density. Given the conditioning information, the implications of a given null model for any conditional moment of interest can be tracked down in the data and compared to the conditional moment implied by the unrestricted SNP density.

It is worth noting that the reprojected density is the density for observed data (i.e., 6month and 5-year yields) estimated from a null model. For some specifications, such as the three factor CIR or affine model, and the two factor regime switching model, the conditional density for yields (under the null model) cannot be characterized by using only two yields (the 6-month, and 5-year yields). Consequently, the reprojected density provides a convenient and comparable benchmark to characterize the conditional density for the two yields under consideration across different null models.

$* * * * * * * * * * * * * * * * * * * * \operatorname{Insert~Figure~} 6$ Here $* * * * * * * * * * * * * * * * * * * *$

For illustrative purposes, Figure 6 plots the reprojected conditional density (evaluated at the sample mean), for the different models under consideration. The unrestricted 6 month yield SNP density has high peak and right tail relative to a normal density with same mean and variance. The unrestricted density for the 5-year yield is skewed to the left. The reprojected densities, evaluated at the sample mean, for the 3-Factor models and the regime shifts model do capture the peakedness to various degrees. The 2-factor regime shift models have greater success in capturing the left skew of the five year yield. ${ }^{15}$

Based on the reprojected densities, Figures 7 and 8 compare the conditional volatility for the various model specifications - this reveals some important differences across models. Note that in the data the process for conditional variance for the 6-month yield is quite different from that of the 5-year yield. The range for the conditional volatility for the 6-month yield rate is much larger than for the 5-year yield - the high end being almost three times the lowest for the 6-month, and two times for the 5-year yield. The short yield volatility is more

\footnotetext{
${ }^{15}$ The shape of the all these conditional distributions will depend on the choice of the conditioning information. For illustrative purposes we have shown the density plot (Figure 6) evaluated at the sample mean. The conditional variance and the conditional covariance dimensions, which are the focus of the remaining section, provide a simple and tractable way to characterize features of this reprojected conditional density across the entire realized sample path of observed yields.
} 
persistent, while the long yield volatility seems more choppy. The 2-Factor[CIR] has difficulty in matching the short rate volatility and does somewhat better in matching the volatility of the 5-year yield. The 2-Factor[RS] model is capable of duplicating the projected volatility of the short rate extremely well, and that of the long yield volatility almost completely. The 3Factor[CIR] and the 3-Factor[AF] model seem to capture the general shape of the volatility much better than the 2-Factor[CIR] model, however, in an absolute sense neither model captures the volatility dynamics as well as the 2-Factor[RS] specification. Also note that the 3-Factor[CIR] specification relative to the affine specification seems to produce greater variation in the volatility, as seen in the data. Finally, the 2-Factor[RSc] model misses the short rate volatility, and its performance is comparable to that of the 3-Factor[CIR] model.

$* * * * * * * * * * * * * * * * * * * *$ Insert Figure 7 Here $* * * * * * * * * * * * * * * * * * * *$

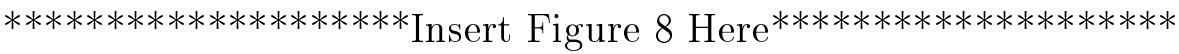

Figure 9 and 10 provide evidence regarding the conditional covariance and correlation between the 6-month and 5-year yield. Again, it is very clear that only the 2-Factor[RS] model succeeds in capturing the wide range of the covariance and correlation observed across these yields. The correlation varies from $40 \%$ to $90 \%$. Note that while the conditional covariance increases during the volatile period of the early 80's, the correlation decreases - suggesting that the volatilities of the two yields rise more rapidly relative to the conditional covariance. The 3-Factor[CIR] and the 3-Factor[AF] specifications have considerable difficulty in capturing the conditional covariance and correlation. However, the 3-Factor[CIR] specification seems to a better job of capturing the conditional covariance relative to the 3 -Factor[AF] specification. The 2-Factor[RS] model comes surprisingly close to capturing virtually all the observed dynamics of the conditional covariance and correlation between these yields. The 2-Factor[RSc] also to a large extent capture the behavior of the the conditional correlation, however, it fails to capture the conditional covariance and short rate volatility. This again highlights the importance of allowing the market price of risk to depend on the regime, as in the preferred 2-Factor[RS] model.

\footnotetext{
$* * * * * * * * * * * * * * * * * * * *$ Insert Figure 9 Here $* * * * * * * * * * * * * * * * * * * *$

$* * * * * * * * * * * * * * * * * * * *$ Insert Figure 10 Here $* * * * * * * * * * * * * * * * * * * *$
} 
Our diagnostics and specification tests show that the model with regime shifts in conjunction with regime dependent market price of risk is important to understand the behavior of the interest rates. In particular, this 2-Factor[RS] structure can account for the conditional volatility and conditional; cross-correlation across yields, along with the conditional higher moments such as skewness and kurtosis.

\section{Concluding Remarks}

There is considerable statistical evidence regarding the presence of regime shifts in the short interest rate data (see Hamilton (1988)). In addition, there are economic reasons as well to believe that interest rates are subject to regime shifts. The transitions between economic expansion and recession have first order effects on the term structure. Despite the potential of important effects from discrete regime shifts, received term structure models, such as the Cox et al. (1985) and affine models, do not incorporate them. The absence of this important component in these models may well explain their poor empirical performance.

The main contribution of this paper is to show that an internally consistent model of the term structure, which incorporates regime shifts, provides significant improvements over multi-factor versions of the CIR and affine models. More specifically, we develop and estimate a model for the term structure which permits regime shifts in both the state vector and the risk premium. We show, that a model which incorporates regime shifts is essential to account for the conditional joint dynamics (i.e., the conditional distribution) of short and long yields. For comparison, we also estimate benchmark multi-factor CIR and affine models. Our empirical exercise is conducted by relying on nominal U.S. treasury bill and bond yields from 1964-1995. For estimation and specification tests of the various models we use the Efficient Method of Moments estimation technique developed in Bansal et al. (1995) and Gallant and Tauchen (1996). We also provide a battery of diagnostics to evaluate the various model specifications, in particular, we rely on the reprojection method to recover the conditional density of yields (conditional on lagged yields) and to evaluate the merits of the different model specifications.

Our empirical evidence shows that benchmark Cox-Ingersoll-Ross term structure models and affine specifications (see Dai and Singleton (2000b)), with up to three factors are sharply rejected in the data - these models cannot jointly account for the violations of the expectations hypothesis, conditional volatility and the conditional cross-correlation across yields 
observed in the data. A two factor regime switching model with regime-dependent risk premium finds considerable support in the data and does surprisingly well in accounting for the violations of the expectations hypothesis, the conditional volatility and cross-correlation of the short and long yields. Further, the absolute pricing error in the cross-section of yields is the smallest for the two factor regime-switching model with regime-dependent risk premium. This preferred model seems to capture the conditional distribution of yields fairly well. Our evidence shows that there is an intimate link between business cycles and regimes extracted from our term structure model-agents in the economy anticipate these discrete regime-shifts and these anticipations are reflected in the time-series and cross-sectional behavior of the yield curves. An empirically tractable equilibrium model that provides direct links to real GDP growth, unemployment, and monetary policy, with regime shifts would be an interesting and valuable extension of this paper. 


\section{Appendix A. General Equilibrium Model}

In this section we provide a general equilibrium model which motivates the pricing kernel and the regime shifts model used in the paper. Consider a standard Lucas (1978) tree economy, with a representative agent whose period utility function is log of consumption. This General Equilibrium economy is discussed in considerable detail in Sargent (1987). It is well recognized that the Intertemporal Marginal Rate of Substitution (IMRS) for this economy (with $0<\beta<1$ ) is

$$
M_{t+1}=\beta \frac{C_{t}}{C_{t+1}}
$$

where $C_{t}$ in equilibrium equals the aggregate exogenous endowment. Consider the value of a claim that delivers the consumption stream $C_{t+s}$ for all $s>0$ - the Euler condition for this asset is

$$
\frac{V_{t}}{C_{t}}=\beta E_{t}\left[\frac{V_{t+1}+C_{t+1}}{C_{t+1}}\right]
$$

Here $V_{t}$ refers to the value of the claim. The above equation implies (see Sargent (1987), page 96, equation 3.13) that $\frac{V_{t}}{C_{t}}=\frac{\beta}{1-\beta}$, the consumption-wealth ratio is constant. The ex post return on the asset that delivers the consumption stream $R_{c, t+1}=\frac{V_{t+1}+C_{t+1}}{V_{t}}=\frac{1}{M_{t+1}}=$ $\left[\beta C_{t} / C_{t+1}\right]^{-1}$, which follows from exploiting the result that $\frac{V_{t}}{C_{t}}=\frac{\beta}{1-\beta}$. It is important to note that this result does not depend on specific assumptions regarding the process for the consumption growth rates. In addition, note that all the above arguments also go through in exactly an analogous manner in a nominal model. In this case the IMRS is defined in an analogous manner with the variable $C_{t}=c_{t} P_{t}$, where $c_{t}$ is the real consumption level and $P_{t}$ the dollar price per unit of consumption good (see Sargent (1987), chapter 5). In the discussion below we will simply refer to the growth of $C_{t}$ as consumption growth.

Hence it follows that

$$
M_{t+1}=\exp \left\{-r_{c, t+1}\right\}
$$

where $r_{c, t+1}=\log \left(R_{c, t+1}\right)$. We will talk about the pricing kernel in terms of the process for $r_{c, t+1}$, as based on the above results, this is equivalent to characterizing the consumption growth rate process. Now assume that $r_{c, t+1}=x_{t}+\frac{\lambda}{\sigma} \frac{x_{t}}{2}+\sqrt{x_{t}} \frac{\lambda}{\sigma} u_{t+1}$, where $x_{t}$ follows the latent square-root process for the 1-Factor[CIR] model (1) considered in the paper. Given this process, the pricing kernel $M_{t+1}$ (as stated in equation (2)) for the CIR case immediately follows. 
Now consider the regime-shifts version of the above model. Again, the above link considers the following latent process as driving the $r_{c, t+1}$ process. Let

$$
\begin{aligned}
& r_{c, t+1}=x_{t}+\left(\frac{\lambda_{0}}{\sigma_{0}}\right)^{2} \frac{x_{t}}{2}+\sqrt{x_{t}} \frac{\lambda_{0}}{\sigma_{0}} u_{t+1} \text { if } s_{t+1}=0, \\
& r_{c, t+1}=x_{t}+\left(\frac{\lambda_{1}}{\sigma_{1}}\right)^{2} \frac{x_{t}}{2}+\sqrt{x_{t}} \frac{\lambda_{1}}{\sigma_{1}} u_{t+1} \text { if } s_{t+1}=1 .
\end{aligned}
$$

The agent at date $t$ only knows $x_{t}$ and $s_{t}$ (which is 0 or 1 ). The agent faces regime uncertainty, which we interpret to economically capture the idea of Hamilton (1989) that the aggregate economy may be subject to persistent regime-shifts (such as due to business cycles) which affects the distribution of the return $r_{c, t+1}$, or equivalently the distribution of consumption growth. Hence, the return $r_{c, t+1}$ (or consumption growth) is a conditional normal with distribution $\mathrm{N}\left(x_{t}+\left(\frac{\lambda_{0}}{\sigma_{0}}\right)^{2} \frac{x_{t}}{2},\left(\frac{\lambda_{0}}{\sigma_{0}}\right)^{2} x_{t}\right)$, if $s_{t+1}=1$, and the distribution is $\mathrm{N}\left(x_{t}+\left(\frac{\lambda_{1}}{\sigma_{1}}\right)^{2} \frac{x_{t}}{2},\left(\frac{\lambda_{1}}{\sigma_{1}}\right)^{2} x_{t}\right)$ if $s_{t+1}=0$. The probability of the future regimes being $s_{t+1}=0$ is $\pi_{i 0}$ and that of $s_{t+1}=1$ is $1-\pi_{i 0}$, where the current regime $s_{t}=i$ (with $i=0,1$ ). The parameter $\lambda_{s_{t+1}}$ affects the volatility (and drift) of the consumption growth in regime $s_{t+1}$.

The process for $x_{t}$ in the regime shifts case is governed by (4) as stated earlier. The above assumptions regarding the regime shifts evolution of $r_{c, t+1}$, lead to the regime shifts pricing kernel in equation (5) discussed in Section 1. Specifically,

$$
\begin{aligned}
& M_{t+1}=\exp \left\{-\left(x_{t}+\left(\frac{\lambda_{0}}{\sigma_{0}}\right)^{2} \frac{x_{t}}{2}+\sqrt{x_{t}} \frac{\lambda_{0}}{\sigma_{0}} u_{t+1}\right)\right\} \text { if } s_{t+1}=0, \\
& M_{t+1}=\exp \left\{-\left(x_{t}+\left(\frac{\lambda_{1}}{\sigma_{1}}\right)^{2} \frac{x_{t}}{2}+\sqrt{x_{t}} \frac{\lambda_{1}}{\sigma_{1}} u_{t+1}\right)\right\} \text { if } s_{t+1}=1 .
\end{aligned}
$$

The probability of regime $s_{t+1}$ is determined by the current regime $s_{t}$ and the regime transition probability matrix $\Pi$ (see equation (3)). The process of $x$ is as stated in equation (4). The above argument gives rise to the regime shifts pricing kernel stated in equation (5). As in the CIR case, this pricing kernel also satisfies the no-arbitrage conditions discussed in Hansen and Jagannathan (1991). As shown below, this pricing kernel also satisfies the condition that $E_{t}\left[M_{t+1}\right]=\exp \left(-r_{f, t}\right) \equiv \exp \left(-x_{t}\right)$, as it should.

Let $P(t, n)$ be the price at date $t$, of a pure discount bond with $n$ periods to maturity. To solve for bond prices we exploit the Euler condition (pricing condition) (see Lucas (1978) and Sargent (1987))

$$
P(t, n)=E\left[M_{t+1} P(t+1, n-1) \mid x_{t}, s_{t}\right]
$$

These are standard first order conditions for valuation. The expression $E\left[M_{t+1} P(t+1, n-\right.$ 1) $\left.\mid x_{t}, s_{t}\right]$ refers to the conditional mean of $M_{t+1} P(t+1, n-1)$ given the information set $x_{t}$ 
and $s_{t}$ at date $t$. Bond prices are measurable with respect to the information set of the agent. Equation (A8), also implies that risk premiums on bond returns are determined by

$\operatorname{cov}_{t}\left[-\frac{M_{t+1}}{E_{t}\left(M_{t+1}\right)}, h_{n, t+1}\right]$, where $h_{n, t+1}$ is the bond return on a $n$ period bond. In the particular context of the regime switching model we will conjecture that $P(t, n)=P_{i}(t, n)$ for regime $s_{t}=i, i=0,1$, for all $n$ and $t$ (see Appendix $\mathrm{C}$ below) and derive an (approximate) analytical solution for bond prices which will solve the Euler equation stated in (A8).

\section{Appendix B. Benchmark CIR Model}

Given the conjectured bond price function $P(t, n)=\exp \left\{-A(n)-B(n) x_{t}\right\}$, no-arbitrage conditions imply that the instantaneous value of a dollar should be one, hence for $n=0$, $A(0)=B(0)=0$. In addition, we also conjecture that $A(1)=0$ and $B(1)=1$, that is $r_{f, t}=x_{t}$. Based on this conjecture, and the assumed process for $x_{t}$, the continuous bond return $\log \left[\frac{P(t+1, n-1)}{P(t, n)}\right]$, is conditionally normally distributed with mean

$$
\mu_{n, t}=-A(n-1)-B(n-1) E_{t}\left[x_{t+1}\right]+A(n)+B(n) x_{t}
$$

and variance

$$
\sigma_{n, t}^{2}=B(n-1)^{2} \operatorname{Var}_{t}\left(x_{t+1}\right)
$$

Exploiting the asset pricing condition, $E_{t}\left[M_{t+1} h_{n, t+1}\right]=1$, the joint lognormality of $M_{t+1}$ and $h_{n, t+1}$, and our conjectured solution, we derive

$$
\exp \left\{-r_{f, t}+\mu_{t, n}+\frac{\sigma_{n, t}^{2}}{2}+B(n-1) \lambda x_{t}\right\}=1 .
$$

The third term in this expression, $\frac{\sigma_{n, t}^{2}}{2}$, is the Jensen's adjustment for continuous returns and the last term, $B(n-1) \lambda x_{t}$ is the risk-premium associated with the bond return. Using the restriction $r_{f, t}=x_{t}$, the definitions for $\mu_{t, n}$, and $\sigma_{t, n}^{2}$, and taking log of (B11) it follows that

$$
-x_{t}-A(n-1)-B(n-1) E_{t}\left[x_{t+1}\right]+A(n)+B(n) x_{t}+\frac{\sigma_{n, t}^{2}}{2}+B(n-1) \lambda x_{t}=0
$$

Equation (B12) says that expected excess returns, in equilibrium, must equal the compensation for systematic risk. Note that the exposure of the current $n$ period bond return to $u_{t+1}$ is $-B(n-1) \sigma \sqrt{x_{t}}$, hence the covariance between the bond return and the $\frac{-M_{t+1}}{E_{t}\left[M_{t+1}\right]}$ equals $-B(n-1) \sigma \sqrt{x_{t}} \times \sqrt{x_{t}} \frac{\lambda}{\sigma}$, i.e., the bond return risk premium is $-B(n-1) \lambda x_{t}$. 
As all of the above arguments are also satisfied for the multi-factor case, for brevity, we report the solutions only for the multi-factor case. With $K$ factors the solutions are

$$
\begin{aligned}
B_{k}(n) & =\left(1-\lambda_{k}-\kappa_{k}\right) B_{k}(n-1)-\frac{1}{2} \sigma_{k}^{2} B_{k}^{2}(n-1)+1, \\
A(n) & =A(n-1)+\sum_{k=1}^{K} \kappa_{k} \theta_{k} B_{k}(n-1),
\end{aligned}
$$

with boundary conditions $A(0)=B_{1}(0)=\cdots=B_{K}(0)=0$. The solution for $A(n)$ and $B_{k}(n)$, implied by (B14), satisfies the asset pricing conditions and is consistent with our conjectured solution for bond prices. This solution is comparable to the continuous time CIR model. Note that the yield-to-maturity of a discount bond in discrete time is defined as

$$
Y(t, n)=-\frac{\ln P(t, n)}{n}=\frac{A(n)}{n}+\sum_{k=1}^{K} \frac{B_{k}(n) x_{k t}}{n} .
$$

Of course, this can be viewed as a special case of the regime shifts model of bond yields (10).

\section{Appendix C. Regime Switching Model}

All the bond risk premia and prices derived below depend on $s_{t}$ and $x_{t}$. To derive the risk-premium restrictions in this section we will make repeated use of the law of iterated expectations. In using the law of iterated expectations we will first form expectations using the "bigger" information set $I_{t+1}=\left\{s_{t+1}, x_{t}, s_{t}\right\}$ and then condition the expectations down to the "smaller" and current information set which contains only $x_{t}$ and $s_{t}$. Note that the "bigger" information set is the "smaller" information set plus information regarding $s_{t+1}$.

Using (5) it follows that $E\left[M_{t+1} \mid x_{t}, s_{t}\right]=\exp \left(-r_{f, t}\right)$ - the price of a one period pure discount bond. To see this, we exploit the law of iterated expectations given that $s_{t}=i$,

$$
E\left[E\left(M_{t+1} \mid I_{t+1}\right) \mid x_{t}, s_{t}\right]=\exp \left(-r_{f, t}\right) E\left[\left(\exp \left\{-\left(\frac{\lambda_{s_{t+1}}}{\sigma_{s_{t+1}}}\right)^{2} \frac{x_{t}}{2}-\frac{\lambda_{s_{t+1}}}{\sigma_{s_{t+1}}} \sqrt{x_{t}} u_{t+1}\right\} \mid I_{t+1}\right) \mid x_{t}, s_{t}\right]
$$

As the conditional mean, $E\left[\exp \left\{-\left(\frac{\lambda_{s_{t+1}}}{\sigma_{s_{t+1}}}\right)^{2} \frac{x_{t}}{2}-\frac{\lambda_{s_{t+1}}}{\sigma_{s_{t+1}}} \sqrt{x_{t}} u_{t+1}\right\} \mid I_{t+1}\right]=\exp (0)$ for $s_{t+1}=0,1$ and the probabilities sum to one- the result follows.

The asset pricing condition that pure discount bond returns, $h_{n, t+1}$, must satisfy the Euler equation, (A8). The pricing condition $E\left[M_{t+1} h_{n, t+1} \mid x_{t}, s_{t}\right]=1$, using law of iterated expectations can be re-stated as,

$$
E\left[E\left(M_{t+1} h_{n, t+1} \mid I_{t+1}\right) \mid x_{t}, s_{t}\right]=\sum_{s_{t+1}=0,1} \pi_{i_{t+1}} E\left[\left(M_{t+1} h_{n, t+1} \mid I_{t+1}\right)\right]=1
$$


To solve for this we need to first characterize the return $h_{n, t+1}$. We conjecture that the bond price with $n$ periods to maturity, at date $t$ depends on the regime $s_{t}=i, i=0,1$, and $x_{t}$ - note that the bond prices are depend only on the current information of the agent, as they should,

$$
P_{i}(t, n)=\exp \left\{-A_{i}(n)-B_{i}(n) x_{t}\right\}
$$

The one period ahead bond price, analogously depends on $s_{t+1}$ and $x_{t+1}$

$$
P_{s_{t+1}}(t+1, n-1)=\exp \left\{-A_{s_{t+1}}(n-1)-B_{s_{t+1}}(n-1) x_{t+1}\right\}
$$

Hence, the continuous one period ahead bond return, that is $\log \left(h_{n, t+1}\right)$ with current regime $s_{t}=i$ equals

$$
\log \left[\frac{P_{s_{t+1}}(t+1, n-1)}{P_{i}(t, n)}\right]=-A_{s_{t+1}}(n-1)-B_{s_{t+1}}(n-1) x_{t+1}+A_{i}(n)+B_{i}(n) x_{t} .
$$

Given $x_{t}, s_{t+1}$, and $s_{t}$, the continuous bond return is conditionally normally distributed with mean

$$
\begin{aligned}
\mu_{n, s_{t+1}, t}= & -A_{s_{t+1}}(n-1)-B_{s_{t+1}}(n-1) E\left[x_{t+1} \mid I_{t+1}\right] \\
& +A_{i}(n)+B_{i}(n) x_{t}
\end{aligned}
$$

and conditional variance

$$
\sigma_{n, s_{t+1}, t}^{2}=B_{s_{t+1}}(n-1)^{2} \sigma_{s_{t+1}}^{2} x_{t}
$$

Note (C22) follows, as $-\sigma_{s_{t+1}} B_{s_{t+1}}(n-1) \sqrt{x_{t}}$ is the exposure of the bond return to the standardized shock $u_{t+1}$ in regime $s_{t+1}$.

For given $x_{t}, s_{t+1}$, and $s_{t}$, the process $M_{t+1} h_{n, t+1}$ is conditionally log-normal, it follows from the asset pricing condition (C17) that

$$
\begin{aligned}
1 & =E\left[M_{t+1} h_{n, t+1} \mid x_{t}, s_{t}\right] \equiv \sum_{s_{t+1}=0,1} \pi_{i s_{t+1}} E\left[M_{t+1} h_{n, t+1} \mid I_{t+1}\right] \\
& =\sum_{s_{t+1}=0,1} \pi_{i s_{t+1}} \exp \left\{-r_{f, t}+\mu_{n, s_{t+1}, t}+\frac{\sigma_{n, s_{t+1}, t}^{2}}{2}+B_{s_{t+1}}(n-1) \lambda_{s_{t+1}} x_{t}\right\}
\end{aligned}
$$

Using the log-linear approximation $\exp (y)-1 \approx y$, and $r_{f, t}=x_{t}$, (C23) leads to the main restriction on the risk premium,

$$
\sum_{s_{t+1}=0,1} \pi_{i s_{t+1}}\left[-x_{t}+\mu_{n, s_{t+1}, t}+\frac{\sigma_{n, s_{t+1}, t}^{2}}{2}+B_{s_{t+1}}(n-1) \lambda_{s_{t+1}} x_{t}\right]=0
$$


with $i=0,1$. The last equation is the key asset pricing restriction (equation (6)) discussed in the main body of the text. Note that the current regime $i$ affects $\mu_{n, s_{t+1}, t}, \sigma_{n, s_{t+1}, t}^{2}$ and the probabilities used in the last equation. Consequently, for each $s_{t}=i$ (with $i=0,1$ ) the analog for the last equation must hold.

The last equation can be rewritten, exploiting the fact that $x_{t}=r_{f, t}$, as

$$
\sum_{s_{t+1}=0,1} \pi_{i s_{t+1}}\left[\mu_{n, s_{t+1}, t}+\frac{\sigma_{n, s_{t+1}, t}^{2}}{2}-r_{f, t}\right]=-\sum_{s_{t+1}=0,1} \pi_{i s_{t+1}}\left[B_{s_{t+1}}(n-1) \lambda_{s_{t+1}} x_{t}\right]
$$

In the above expression the future regime dependence $s_{t+1}$ is integrated out. Hence, the risk premium $-\sum_{s_{t+1}=0,1} \pi_{i s_{t+1}}\left[B_{s_{t+1}}(n-1) \lambda_{s_{t+1}} x_{t}\right] \equiv-x_{t} E\left[B_{s_{t+1}}(n-1) \lambda_{s_{t+1}} \mid s_{t}\right]$ is measurable with respect to the current information set of the agent and depends only on $s_{t}$ and $x_{t}$. The risk premium is determined by the conditional covariance between the $-M_{t+1} / E_{t}\left[M_{t+1}\right]$, and the asset return. Hence the product of the exposure of $-M_{t+1} / E_{t}\left[M_{t+1}\right]$ to $u_{t+1}$ in regime $s_{t+1}$, with that of the exposure of the asset return in the regime determines the risk compensation for that regime. In particular, this product, as discussed in Section 1 of the paper is,

$$
-\sigma_{s_{t+1}} B_{s_{t+1}}(n-1) \sqrt{x_{t}} \times\left[\frac{\lambda_{s_{t+1}}}{\sigma_{s_{t+1}}} \sqrt{x_{t}}\right]=-B_{s_{t+1}}(n-1) \lambda_{s_{t+1}} x_{t}
$$

The risk premium on the bond at date $t$ is then determined by averaging over $s_{t+1}$ using the transition probabilities for $s_{t+1}$ given that the agent knows $s_{t}$. This leads to the risk premium expression $-x_{t} E\left[B_{s_{t+1}}(n-1) \lambda_{s_{t+1}} \mid s_{t}\right]$. Note as in the CIR case, when an asset return has an unit standard deviation exposure to the $u_{t+1}$ shock (recall $u$ has unit volatility) - then the above restriction also tells us about the market price of risk for this shock (see Cochrane (2001)). An asset which has -1 exposure to the $u_{t+1}$ shock will have an risk-premium of $-E\left[\frac{\lambda_{s_{t+1}}}{\sigma_{t+1}} \mid s_{t}\right] \sqrt{x_{t}}$ - this is the market price of risk for this shock in the regime shifts economy, as discussed in Section 1 of the paper.

The above risk premium restriction is all that is needed to solve for the unknown coefficients $A_{s}$ and $B_{s}$ to derive the bond prices - that is for any current regime $s_{t}=i=0,1$, we must have

$$
\begin{aligned}
\sum_{s_{t+1}=0,1} \pi_{0 s_{t+1}}\left[\mu_{n, s_{t+1}, t}+\frac{\sigma_{n, s_{t+1}, t}^{2}}{2}-r_{f, t}\right] & =-\sum_{s_{t+1}=0,1} \pi_{0 s_{t+1}}\left[B_{s_{t+1}}(n-1) \lambda_{s_{t+1}} x_{t}\right] \\
\sum_{s_{t+1}=0,1} \pi_{1 s_{t+1}}\left[\mu_{n, s_{t+1}, t}+\frac{\sigma_{n, s_{t+1}, t}^{2}}{2}-r_{f, t}\right] & =-\sum_{s_{t+1}=0,1} \pi_{1 s_{t+1}}\left[B_{s_{t+1}}(n-1) \lambda_{s_{t+1}} x_{t}\right]
\end{aligned}
$$


The above analysis is also satisfied for the case of multiple continuous factors $x_{k t}$, and a given regime shifts process, with regime $s_{t}=0,1$. For brevity we report the solution for the case of two continuous factors with regime shifts, for factor $k=1,2$, as

$$
\left[\begin{array}{c}
B_{k 0}(n) \\
B_{k 1}(n)
\end{array}\right]=\left[\begin{array}{ll}
\pi_{00} & \pi_{01} \\
\pi_{10} & \pi_{11}
\end{array}\right]\left[\begin{array}{c}
\left(1-\kappa_{k 0}-\lambda_{k 0}\right) B_{k 0}(n-1)-\frac{1}{2} \sigma_{k 0}^{2} B_{k 0}^{2}(n-1)+1 \\
\left(1-\kappa_{k 1}-\lambda_{k 1}\right) B_{k 1}(n-1)-\frac{1}{2} \sigma_{k 1}^{2} B_{k 1}^{2}(n-1)+1
\end{array}\right],
$$

and

$$
\left[\begin{array}{l}
A_{0}(n) \\
A_{1}(n)
\end{array}\right]=\left[\begin{array}{ll}
\pi_{00} & \pi_{01} \\
\pi_{10} & \pi_{11}
\end{array}\right]\left[\begin{array}{l}
A_{0}(n-1)+\kappa_{10} \theta_{10} B_{10}(n-1)+\kappa_{20} \theta_{20} B_{20}(n-1) \\
A_{1}(n-1)+\kappa_{11} \theta_{11} B_{11}(n-1)+\kappa_{21} \theta_{21} B_{21}(n-1)
\end{array}\right],
$$

with initial conditions (no arbitrage conditions) that $A_{0}(0)=A_{1}(0)=B_{10}(0)=B_{11}(0)=$ $B_{20}(0)=B_{21}(0)=0$.

\section{Appendix D. SNP Density and EMM Estimation}

We now provide technical details on (a) how to construct a semi-nonparametric (SNP) density to generate the data-dependent moment conditions, and (b) how to estimate the structural parameters of a term structure model by matching the SNP scores with long simulation data via EMM.

Following Gallant and Tauchen (1996), any smooth conditional density function can be approximated arbitrarily close by a Hermite polynomial expansion. Let $y$ be the vector of the interest rates under consideration, $x$ be the vector of lagged $y$. The auxiliary $f$-model has a density function defined by a modified Hermite polynomial,

$$
f(y \mid x, \theta)=C\left\{[\mathcal{P}(z, x)]^{2} \phi\left(y \mid \mu_{x}, \Sigma_{x}\right)\right\}
$$

where $\mathcal{P}$ is a polynomial with degree $K_{z}$ in $z$, which is a standardized transformation $z=$ $R_{x}^{-1}\left(y-\mu_{x}\right)$ with $\Sigma_{x}=R_{x} R_{x}^{\prime}$. The square of $\mathcal{P}$ makes the density positive and the argument of the polynomial is $z$. The coefficients of the polynomial are allowed to be another polynomial of degree $K_{x}$ in $x$. The constant in the polynomial of $z$ is set to 1 for identification. $C$ is a normalizing factor to make the density integrating to one and $\phi(\cdot)$ is a normal density of $y$ with conditional mean $\mu_{x}$ and conditional variance $\Sigma_{x}$, where $\mu_{x}$ is estimated by using a VAR specification, and $\Sigma_{x}$ is estimated by using an ARCH specification, which parameterizes $R_{x}$. Note that both $\mu_{x}$ and $R_{x}$ depend only on lags of $y$, that is the vector $x$. 
The length of the auxiliary model (i.e., the SNP density) parameter is determined by the number of lags in the VAR mean specification $L_{\mu}$, lags in the ARCH specification $L_{r}$, lags of $x$ used in constructing the coefficients of the polynomial $L_{p}$, the degree $K_{z}$ of the polynomial in $z$, and the degree $K_{x}$ of the polynomial in $x$. The specific choice for these tuning parameters, and consequently the SNP conditional density is done by relying on the BIC information criterion. Table A1 bellow explains how the tuning parameters are linked to the SNP specification.

\section{***********************Insert Table A1 Here $* * * * * * * * * * * * * * * * * * * *$}

Let $\left\{\tilde{y}_{t}\right\}_{t=1}^{n}$ be the observed data, and $\tilde{x}_{t-1}$ be the lagged observations. The sample mean $\log$ likelihood function is defined by $\mathcal{L}_{n}\left(\theta,\left\{\tilde{y}_{t}\right\}_{t=1}^{n}\right)=\frac{1}{n} \sum_{t=1}^{n} \log \left[f\left(\tilde{y}_{t} \mid \tilde{x}_{t-1}, \theta\right)\right]$, where $\theta$ are the unknown parameters of this conditional density, which need to be estimated. A quasi-maximum-likelihood estimator is obtained by

$$
\tilde{\theta}_{n}=\arg \max _{\theta} \mathcal{L}_{n}\left(\theta,\left\{\tilde{y}_{t}\right\}_{t=1}^{n}\right)
$$

The dimension of the auxiliary $f$-model, the length of $\theta$, is selected by the Schwarz's Bayesian Information Criterion (BIC) $B I C=s_{n}(\tilde{\theta})+\frac{l_{\theta}}{2 n} \log (n)$, where $s_{n}(\tilde{\theta})=-\mathcal{L}_{n}\left(\tilde{\theta},\left\{\tilde{y}_{t}\right\}_{t=1}^{n}\right)$ is the negative maximized objective function and $l_{\theta}$ is the length of the auxiliary model. For greater details regarding SNP see Gallant and Tauchen (1989).

From the first stage seminonparametric estimates one obtained the fitted scores as the moment conditions, $m_{n}(\tilde{\theta})=\frac{1}{n} \sum_{t=1}^{n} \frac{\partial}{\partial \theta} \log f\left(\tilde{y}_{t} \mid \tilde{x}_{t-1}, \tilde{\theta}\right)$. In the second stage, a SMM-type estimator is implemented in the following way. Let $\left\{\hat{y}_{t}\right\}_{t=1}^{N}$ be a long simulation from a candidate value of $\rho$, the parameter vector of the maintained structural model. The auxiliary score functions can be re-evaluated at the simulated data, $\hat{m}_{N}(\rho, \tilde{\theta})=\frac{1}{N} \sum_{t=1}^{N} \frac{\partial}{\partial \theta} \log f\left(\hat{y}_{t} \mid \hat{x}_{t-1}, \tilde{\theta}\right)$, and the minimum chi-square estimator is simply minimizing the quadratic objective function,

$$
\hat{\rho}_{n}=\arg \min _{\rho}\left\{\hat{m}_{N}(\rho, \tilde{\theta})^{\prime} \tilde{\mathcal{I}}^{-1} \hat{m}_{N}(\rho, \tilde{\theta})\right\}
$$

where the weighting matrix $\tilde{\mathcal{I}}^{-1}$ is estimated by the mean-outer-product of SNP scores $\tilde{\mathcal{I}}_{n}=\frac{1}{n} \sum_{t=1}^{n}\left[\frac{\partial}{\partial \theta} \log f\left(\tilde{y}_{t} \mid \tilde{x}_{t-1}, \tilde{\theta}\right)\right]\left[\frac{\partial}{\partial \theta} \log f\left(\tilde{y}_{t} \mid \tilde{x}_{t-1}, \tilde{\theta}\right)\right]^{\prime}$. Under regularity conditions on the data generating process, one can show that the estimator is almost surely convergent and asymptotically normal. The asymptotic variance can be estimated by its empirical counterpart,

$$
\widehat{V A R}(\hat{\rho})=\frac{1}{n}\left[\left(\hat{M}_{n}\right)^{\prime}\left(\tilde{I}_{n}\right)^{-1}\left(\hat{M}_{n}\right)\right]^{-1},
$$


where $M(\rho, \theta)=\left(\partial / \partial \rho^{\prime}\right) m(\rho, \theta)$ are the gradients of moment conditions. The normalized criterion function value in the EMM estimation forms a specification test for the overidentifying restrictions

$$
n \hat{m}_{N}(\rho, \tilde{\theta})^{\prime} \tilde{\mathcal{I}}^{-1} \hat{m}_{N}(\rho, \tilde{\theta}) \sim \mathcal{X}^{2}\left(l_{\theta}-l_{p}\right),
$$

with the degree of freedom equals $l_{\theta}-l_{p}$, the number of scores (i.e., moment conditions) in the auxiliary model, $l_{\theta}$, less the number of structural parameters, $l_{p}$. It is assumed that $l_{p}$ is smaller than $l_{\theta}$. 


\section{References}

Ahn, Dong-Hyun, Robert F. Dittmar, and A. Ronald Gallant (2001), "Quadratic Term Structure Models: Theory and Evidence," Review of Financial Studies, forthcoming.

Andersen, Torben G., Hyung-Jin Chung, and Bent E. Sørenson (1999), "Efficient Method of Moments Estimation of a Stochastic Volatility Model: A Monte Carlo Study," Journal of Econometrics, vol. 91, 61-87.

Ang, Andrew and Geert Bekaert (1998), "Regime Switches in Interest Rates," Working Paper 6508, NBER, Cambridge, MA.

Backus, David, Silverio Foresi, Abon Mozumdar, and Liuren Wu (2000), "Predictable Changes in Yields and Forward Rates," Working Paper.

Backus, David and Stanley Zin (1994), "Reverse Engineering the Yield Curve," Working Paper 4676, NBER, Cambridge, MA.

Bansal, Ravi and Wilbur John Coleman (1996), "A Monetary Explanation of the Equity Premium, Term Premium, and Risk-Free Rate Puzzles," Journal of Political Economy, vol. 104, 1135-1171.

Bansal, Ravi, A. Ronald Gallant, Robert Hussey, and George Tauchen (1995), "Nonparametric Estimation of Structural Models for High-Frequency Currency Market Data," Journal of Econometrics, vol. 66, 251-287.

Bekaert, Geert, Robert J. Hodrick, and David A. Marshall (1997), "On Biases in Tests of the Expectation Hypothesis of the Term Structure of Interest Rates," Journal of Financial Economics, vol. 44, 309-348.

Brown, Stephen J. and Philip H. Dybvig (1986), "The Empirical Implications of the Cox, Ingersoll, Ross Theory of the Term Structure of Interest Rates," Journal of Finance, vol. 41, 617-630.

Cai, Jun (1994), "A Markov Model of Switching-Regime ARCH," Journal of Business and Economic Statistics, vol. 12, 309-316.

Campbell, John Y., Andrew W. Lo, and A. Craig MacKinlay (1997), The Econometrics of Financial Markets, Princeton University Press,, Princeton, NJ. 
Campbell, John Y. and Robert J. Shiller (1991), "Yield Spreads and Interest Rate Movements: A Bird's Eye View," Review of Economic Studies, vol. 58, 495-514.

Chen, Lin (1996), "Stochastic Mean and Stochastic Volatility - A Three-Factor Model of the Term Structure of Interest Rates and Its Applications in Derivatives Pricing and Risk Management," Financial Markets, Institution and Instruments, vol. 5, 1-88.

Chen, Ren-Raw and Louis Scott (1993), "Maximum Likelihood Estimation for a Multifactor Equilibrium Model of the Term Structure of Interest Rates," Journal of Fixed Income, vol. $3,14-31$.

Cochrane, John H. (2001), Asset Pricing, Princeton University Press,, Princeton, NJ.

Cox, John C., Jonathan E. Ingersoll, and Stephen A. Ross (1985), "A Theory of the Term Structure of Interest Rates," Econometrica, vol. 53, 385-407.

Dai, Qiang (2000), "From Equity Premium Puzzle to Expectations Puzzle: A General Equilibrium Production Economy with Stochastic Habit Formation," Working Paper, Stern School of Business, New York University.

Dai, Qiang and Kenneth J. Singleton (2000a), "Expectation Puzzles, Time-varying Risk Premia, and Dynamic Models of the Term Structure," Working Paper, Stern School of Business, New York University; Graduate School of Business, Stanford University.

Dai, Qiang and Kenneth J. Singleton (2000b), "Specification Analysis of Affine Term Structure Models," Journal of Finance, vol. 55, 1943-1978.

Duarte, Jefferson (1999), "The Relevance of the Parameterization of Price of Risk in Affine Term Structure Models," Working Paper, Graduate School of Business, University of Chicago.

Duffee, Gregory (2000), "Forecasting Future Interest Rates: Are Affine Models Failures?" Working Paper, Haas School of Business, University of California at Berkeley.

Duffie, Darrell and Kenneth Singleton (1993), "Simulated Moments Estimation of Markov Models of Asset Prices," Econometrica, vol. 61, 929-952.

Duffie, Darrell and Kenneth Singleton (1997), "An Econometric Model of the Term Structure of Interest-Rate Swap Yields," Journal of Finance, vol. 52, 1287-1321. 
Engle, Robert F. and Gloria González-Rivera (1991), "Semiparametric ARCH Models," Journal of Business and Economic Statistics, vol. 9, 345-359.

Estrella, Arturo and Gikas A. Hardouvelis (1991), "The Term Structure as a Predictor of Real Economic Activity," Journal of Finance, vol. 46, 555-576.

Evans, Martin D. D. (1998), "Regime Shifts, Risk and the Term Structure," Working Paper, Department of Economics, Georgetown University.

Fama, Eugene F. and Kenneth R. French (1989), "Business Conditions and Expected Returns on Stocks and Bonds," Journal of Financial Economics, vol. 25, 23-49.

Gallant, A. Ronald and George Tauchen (1989), "Seminonparametric Estimation of Conditionally Constrained Heterogeneous Processes: Asset Pricing Applications," Econometrica, vol. 57, 1091-1120.

Gallant, A. Ronald and George Tauchen (1996), "Which Moment to Match?" Econometric Theory, vol. 12, 657-681.

Gallant, A. Ronald and George Tauchen (1998), "Reprojecting Partially Observed Systems with Application to Interest Rate Diffusions," Journal of the American Statistical Association, vol. 93, 10-24.

Gallant, A. Ronald and George Tauchen (1999), "The Relative Efficiency of Method of Moments Estimators," Journal of Econometrics, vol. 92, 149-172.

Garcia, René (1992), "Asymptotic Null Distribution of the Likelihood Ratio Test in Markov Switching Models," Working Paper, université de Montréal.

Garcia, René and Pierre Perron (1996), "An Analysis of the Real Interest Rate Under Regime Shifts," Review of Economics and Statistics, vol. 78, 111-125.

Gibbons, Michael R. and Krishna Ramaswamy (1993), "A Test of the Cox, Ingersoll, and Ross Model of the Term Structure," Review of Financial Studies, vol. 6, 619-658.

Gray, Stephen F. (1996), "Modeling the Conditional Distribution of Interest Rates as A Regime-Switching Process," Journal of Financial Economics, vol. 42, 27-62. 
Hamilton, James D. (1988), "Rational Expectations Econometric Analysis of Changes in Regimes: An Investigation of the Term Structure of Interest Rates," Journal of Economic Dynamics and Control, vol. 12, 385-423.

Hamilton, James D. (1989), "A New Approach to the Economic Analysis of Nonstationary Time Series and the Business Cycle," Econometrica, vol. 57, 357-384.

Hamilton, James D. (1990), "Analysis of Time Series Subject to Changes in Regime," Journal of Econometrics, vol. 45, 39-70.

Hansen, Bruce E. (1992), “The Likelihood Ratio Test under Non-Standard Conditions: Testing the Markov Switching Model of GNP," Journal of Applied Econometrics, vol. 7, $\mathrm{s} 61-\mathrm{s} 82$.

Hansen, Lars Peter and Ravi Jagannathan (1991), "Implications of Security Market Data for Models of Dynamic Economies," Journal of Political Economy, vol. 99, 225-262.

Hansen, Lars Peter and Ravi Jagannathan (1997), "Assessing Specification Errors in Stochastic Discount Factor Models," Journal of Finance, vol. 52, 557-590.

Harvey, Campbell R. (1988), "The Real Term Structure and Consumption Growth," Journal of Financial Economics, vol. 22, 305-333.

Jagannathan, Ravi, Andrew Kaplin, and Guoqiang Sun (2000), "An Evaluation of MultiFactor CIR Models Using LIBOR, Swap Rates, and Cap and Swaption Prices," Working Paper, Kellogg Graduate School of Management, Northwestern University.

Liu, Jun, Francis A. Longstaff, and Ravit E. Mandell (2000), "The Market Price of Credit Risk: An Empirical Analysis of Interest Rate Swap Spread," Working Paper, The Anderson School at UCLA.

Longstaff, Francis A. and Eduardo S. Schwartz (1992), "Interest Rate Volatility and the Term Structure: A Two-Factor General Equilibrium Model," Journal of Finance, vol. 47, 1259-1282.

Lucas, Robert E. (1978), "Asset Prices in an Exchange Economy," Econometrica, vol. 46, 1429-1445. 
Naik, Vasant and Moon Hoe Lee (1997), "Yield Curve Dynamics with Discrete Shifts in Economic Regimes: Theory and Estimation," Working Paper, Faculty of Commerce, University of British Columbia.

Pearson, Neil D. and Tong-Sheng Sun (1994), "Exploiting the Conditional Density in Estimating the Term Structure: An Application to the Cox, Ingersoll, and Ross Model," Journal of Finance, vol. 49, 1279-1304.

Roberds, William and Charlse H. Whiteman (1999), "Endogenous Term Premia and Anomalies in the Term Struture of Interest Rates: Explaining the Predictability Smile," Journal of Monetary Economics, vol. 44, 555-580.

Sargent, Thomas J. (1987), Dynamic Macroeconomic Theory, Harvard University Press,, Cambridge, MA.

Sun, Tong-Sheng (1992), "Real and Nominal Interest Rate: A discrete-Time Model and Its Continuous-Time Limit," Review of Financial Studies, vol. 5, 581-611.

Tauchen, George (1997), "New Minimum Chi-Square Methods in Empirical Finance," in "Advances in Econometrics, Seventh World Congress," (edited by Kreps, D. and K. Wallis), Cambridge University Press, Cambridge UK.

Zhou, Hao (2001), "Finite Sample Properties of EMM, GMM, QMLE, and MLE for a SquareRoot Interest Rate Diffusion Model," Journal of Computational Finance, forthcoming. 
Table 1: Summary Statistics of Monthly Yield Data

There are 379 monthly observations of the yields with nine maturities. The data is obtained from CRSP (Center for Research in Security Prices) bond files, ranging from June 1964 to December 1995.

\begin{tabular}{lrrrrrrrrr}
\hline \hline Maturity & $1 \mathrm{mn}$ & $3 \mathrm{mn}$ & $6 \mathrm{mn}$ & $9 \mathrm{mn}$ & $1 \mathrm{yr}$ & $2 \mathrm{yr}$ & $3 \mathrm{yr}$ & $4 \mathrm{yr}$ & $5 \mathrm{yr}$ \\
\hline Mean & 0.0645 & 0.0672 & 0.0694 & 0.0709 & 0.0713 & 0.0734 & 0.0750 & 0.0762 & 0.0769 \\
Std Dev & 0.0265 & 0.0271 & 0.0270 & 0.0269 & 0.0260 & 0.0252 & 0.0244 & 0.0240 & 0.0237 \\
Skewness & 1.2111 & 1.2118 & 1.1518 & 1.1013 & 1.0307 & 0.9778 & 0.9615 & 0.9263 & 0.8791 \\
Kurtosis & 4.5902 & 4.5237 & 4.3147 & 4.1605 & 3.9098 & 3.6612 & 3.5897 & 3.5063 & 3.3531 \\
Minimum & 0.0265 & 0.0277 & 0.0287 & 0.0299 & 0.0311 & 0.0366 & 0.0387 & 0.0397 & 0.0398 \\
Maximum & 0.1640 & 0.1612 & 0.1655 & 0.1644 & 0.1581 & 0.1564 & 0.1556 & 0.1582 & 0.1500 \\
\hline \hline
\end{tabular}


Table 2: SNP Score Generator

The SNP score generator has a leading ARCH term with $L_{\mu}$ lags in conditional mean, $L_{r}$ in conditional standard deviation. The standardized innovation has a normal density stretched by a squared Hermite polynomial with degree of $K_{z}$. Since it is a bivariate SNP density, the interaction polynomial term above the $I_{z}$ degree is suppressed as zero. Similarly the coefficient of the $z$-polynomial may depend on the lagged history through a $K_{x}$ degree polynomial with the interaction term above $I_{x}$ degree being suppressed as zero. The lag order $L_{p}$ on $x$-polynomial is inoperative if $K_{x}=0$ and set to $L_{p}=1$ by convention. The total number of parameters is $l_{\theta}$. The BIC preferred choice is $\mathrm{s} 1514200$. The last two columns report the minimized objective function

$$
s_{n}(\tilde{\theta})=-\frac{1}{n} \sum_{t=1}^{n} \log \left[f\left(\tilde{y}_{t} \mid \tilde{x}_{t-1}, \tilde{\theta}\right)\right]
$$

and BIC information criterion

$$
B I C=s_{n}(\tilde{\theta})+\frac{l_{\theta}}{2 n} \log (n) .
$$

\begin{tabular}{rrrrrrrrrr}
\hline \hline$L_{\mu}$ & $L_{r}$ & $L_{p}$ & $K_{z}$ & $I_{z}$ & $K_{x}$ & $I_{x}$ & $l_{\theta}$ & $s_{n}(\tilde{\theta})$ & $\mathrm{BIC}$ \\
\hline 1 & 0 & 1 & 0 & 0 & 0 & 0 & 9 & -0.79431 & -0.72073 \\
2 & 0 & 1 & 0 & 0 & 0 & 0 & 13 & -0.82011 & -0.71383 \\
3 & 0 & 1 & 0 & 0 & 0 & 0 & 17 & -0.82763 & -0.68865 \\
\hline 1 & 1 & 1 & 0 & 0 & 0 & 0 & 11 & -0.96388 & -0.87395 \\
1 & 2 & 1 & 0 & 0 & 0 & 0 & 13 & -0.98904 & -0.88276 \\
1 & 3 & 1 & 0 & 0 & 0 & 0 & 15 & -1.01872 & -0.89609 \\
1 & 4 & 1 & 0 & 0 & 0 & 0 & 17 & -1.07256 & -0.93358 \\
1 & 5 & 1 & 0 & 0 & 0 & 0 & 19 & -1.09188 & -0.93656 \\
1 & 6 & 1 & 0 & 0 & 0 & 0 & 21 & -1.09328 & -0.92160 \\
1 & 7 & 1 & 0 & 0 & 0 & 0 & 23 & -1.09922 & -0.91119 \\
\hline 1 & 5 & 1 & 4 & 3 & 0 & 0 & 27 & -1.14488 & -0.92416 \\
1 & 5 & 1 & 4 & 2 & 0 & 0 & 28 & -1.17202 & -0.94311 \\
1 & 5 & 1 & 4 & 1 & 0 & 0 & 30 & -1.17230 & -0.92704 \\
1 & 5 & 1 & 4 & 0 & 0 & 0 & 33 & -1.20102 & -0.93124 \\
1 & 5 & 1 & 5 & 4 & 0 & 0 & 29 & -1.15141 & -0.91433 \\
1 & 5 & 1 & 5 & 3 & 0 & 0 & 30 & -1.17326 & -0.92801 \\
1 & 5 & 1 & 5 & 2 & 0 & 0 & 32 & -1.18655 & -0.92494 \\
1 & 5 & 1 & 5 & 1 & 0 & 0 & 35 & -1.21327 & -0.92714 \\
1 & 5 & 1 & 5 & 0 & 0 & 0 & 39 & -1.21690 & -0.89807 \\
1 & 5 & 1 & 6 & 5 & 0 & 0 & 31 & -1.17734 & -0.92391 \\
1 & 5 & 1 & 6 & 4 & 0 & 0 & 32 & -1.18399 & -0.92239 \\
1 & 5 & 1 & 6 & 3 & 0 & 0 & 34 & -1.18758 & -0.90962 \\
1 & 5 & 1 & 6 & 2 & 0 & 0 & 37 & -1.19769 & -0.89521 \\
1 & 5 & 1 & 6 & 1 & 0 & 0 & 41 & -1.22820 & -0.89302 \\
1 & 5 & 1 & 6 & 0 & 0 & 0 & 46 & -1.23516 & -0.85910 \\
1 & 5 & 1 & 4 & 2 & 1 & 0 & 48 & -1.24496 & -0.85256 \\
1 & 5 & 1 & 4 & 2 & 2 & 1 & 68 & -1.28923 & -0.73332 \\
1 & 5 & 1 & 4 & 2 & 2 & 0 & 78 & -1.30853 & -0.67087 \\
\hline \hline & & & & & & & & & \\
& &
\end{tabular}


Table 3: Parameter Estimates of Projected SNP Density

The SNP density is described in Section 2.1, and the specification search is reported in Table 2. The parameter in the Hermite polynomial function $a(i, j)$ stands for the term with $i$ 'th power on the short yield and $j$ 'th power on the long yield. The parameter in the conditional mean function is respectively $\mu(1,0)$ constant in short yield, $\mu(2,0)$ constant in long yield, $\mu(1,1)$ lag one short yield in short yield equation, $\mu(2,1)$ lag one long yield in short yield equation, $\mu(1,2)$ lag one short yield in long yield equation, and $\mu(2,2)$ lag one long yield in long yield equation. The parameter in ARCH standard deviation function is $R(k, l)$ - the short yield $(k=1)$ or long yield $(k=2)$ with lag equals to $l . R(3)$ is the constant off-diagonal term, a partial contribution to the covariance. The negative sample mean log-likelihood $s_{n}$ and BIC information criterion are the same as those in Table 2.

\begin{tabular}{|c|c|c|}
\hline Parameter & Estimate & Standard Error \\
\hline Hermite $a(0,0)$ & 1.00000 & $(0.00000)$ \\
\hline$a(0,1)$ & -0.03861 & $(0.08103)$ \\
\hline$a(1,0)$ & 0.49154 & $(0.10908)$ \\
\hline$a(0,2)$ & -0.13739 & $(0.06996)$ \\
\hline$a(1,1)$ & 0.17944 & $(0.07162)$ \\
\hline$a(2,0)$ & -0.00415 & $(0.09781)$ \\
\hline$a(0,3)$ & 0.01735 & $(0.01406)$ \\
\hline$a(3,0)$ & -0.06015 & $(0.04077)$ \\
\hline$a(0,4)$ & 0.02044 & $(0.00742)$ \\
\hline$a(4,0)$ & -0.01098 & $(0.01556)$ \\
\hline Mean $\mu(2,0)$ & -0.09681 & $(0.01521)$ \\
\hline$\mu(1,0)$ & -0.02068 & $(0.01273)$ \\
\hline$\mu(2,2)$ & 0.95940 & $(0.02201)$ \\
\hline$\mu(2,1)$ & 0.02563 & $(0.01576)$ \\
\hline$\mu(1,2)$ & -0.01187 & $(0.01954)$ \\
\hline$\mu(1,1)$ & 0.94979 & $(0.01529)$ \\
\hline ARCH $R(1,0)$ & 0.04595 & $(0.01034)$ \\
\hline$R(2,0)$ & 0.08426 & $(0.01093)$ \\
\hline$R(3)$ & 0.19853 & $(0.07145)$ \\
\hline$R(1,1)$ & 0.10511 & $(0.01548)$ \\
\hline$R(2,1)$ & 0.12883 & $(0.04020)$ \\
\hline$R(1,2)$ & -0.01868 & $(0.05845)$ \\
\hline$R(2,2)$ & 0.13730 & $(0.04346)$ \\
\hline$R(1,3)$ & 0.02818 & $(0.06784)$ \\
\hline$R(2,3)$ & 0.07998 & $(0.04836)$ \\
\hline$R(1,4)$ & 0.25753 & $(0.07586)$ \\
\hline$R(2,4)$ & 0.12819 & $(0.05029)$ \\
\hline$R(1,5)$ & 0.03728 & $(0.05829)$ \\
\hline$R(2,5)$ & 0.22468 & $(0.05443)$ \\
\hline $\mathrm{Spec}=\mathrm{s} 1514200$ & $s_{n}=-1.17202$ & $\mathrm{BIC}=-0.94311$ \\
\hline
\end{tabular}




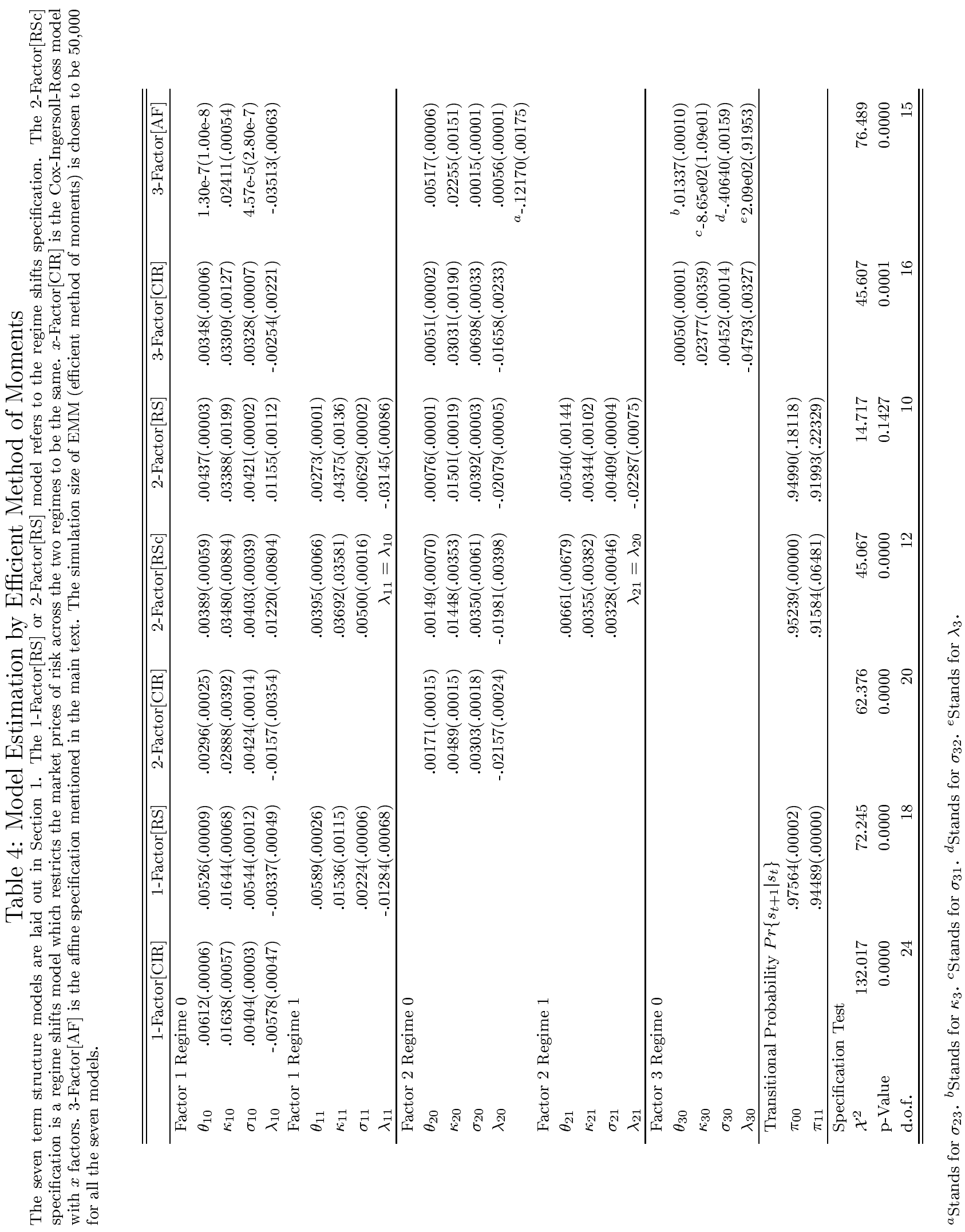




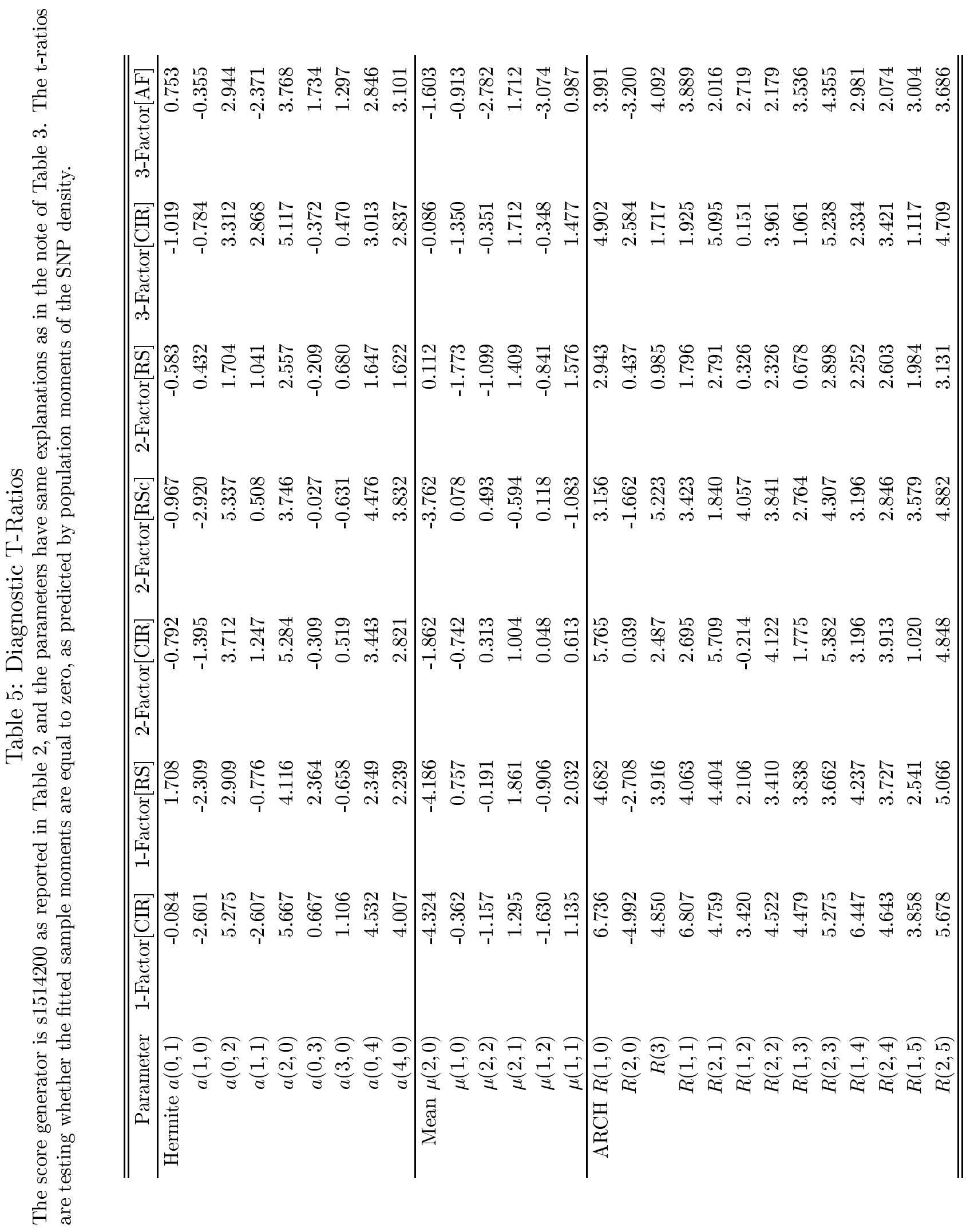




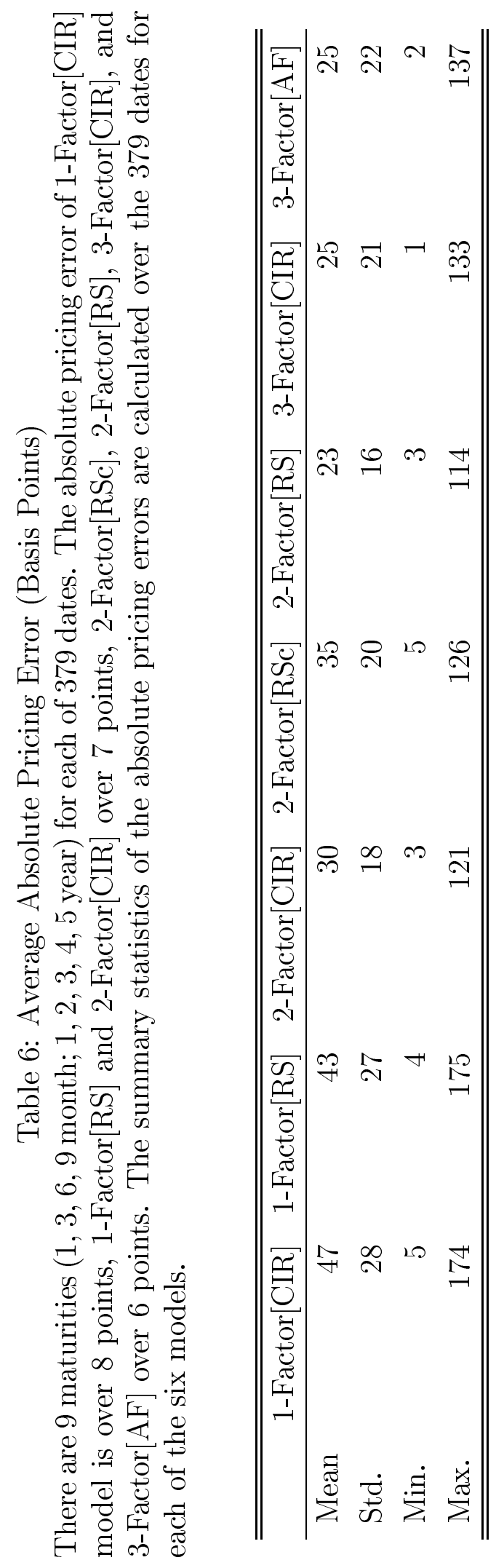


Table 7: Characteristics across Regimes (Basis Points)

Short rate is the 6 month yield and long rate is the 5 year yield. The first difference of interest rates measures the ex post signed volatility. The convexity is calculated using 1 , 2 , and 3 year adjacent yields, and the results are similar for other maturity combinations. The economic-phase regime classification is from the NBER business cycle indicator, and the model-implied regime classification is from minimizing the pricing error of the 2-Factor[RS] model.

\begin{tabular}{lrr}
\hline \hline Characteristics & Economic Expansion Regime & Economic Recession Regime \\
\hline Short Rate Level & 652 & 936 \\
Short Rate First Difference & 5 & -26 \\
Long Rate First Difference & 2 & -8 \\
Yield Spread & 86 & 11 \\
Convexity & -6 & 0 \\
\hline \hline Characteristics & Model Implied Regime 1 & Model Implied Regime 0 \\
\hline Short Rate Level & 691 & 721 \\
Short Rate First Difference & 1 & -6 \\
Long Rate First Difference & 2 & -14 \\
Yield Spread & 83 & -3 \\
Convexity & -8 & 16 \\
\hline \hline
\end{tabular}




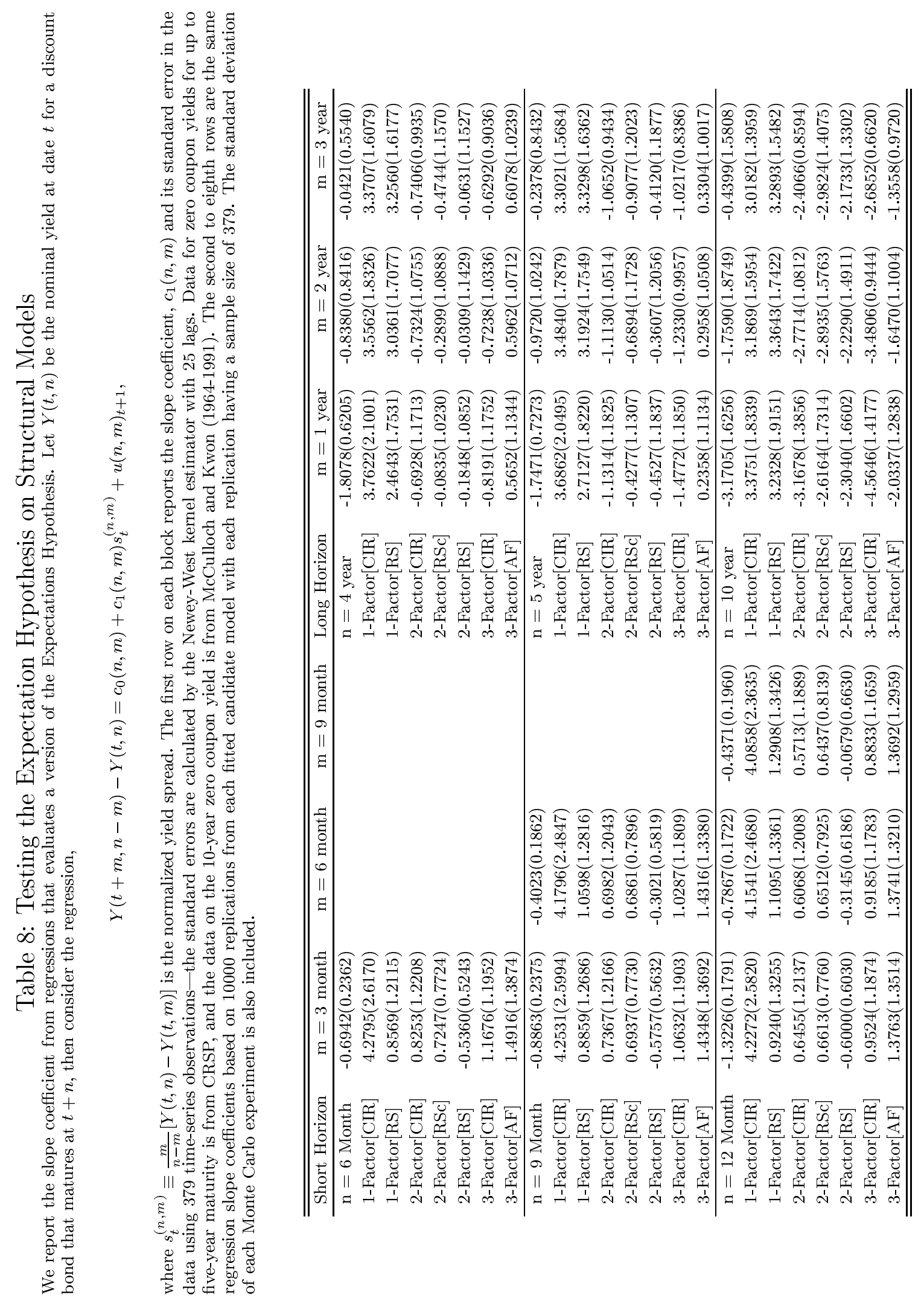


Table A1: Restrictions Implied by Settings of the Tuning Parameters.

\begin{tabular}{ll}
\hline \hline Parameter setting & Characterization of $\left\{y_{t}\right\}$ \\
\hline$L_{u}=0, L_{r}=0, L_{p} \geq 0, K_{z}=0, K_{x}=0$ & iid Gaussian \\
$L_{u}>0, L_{r}=0, L_{p} \geq 0, K_{z}=0, K_{x}=0$ & Gaussian VAR \\
$L_{u}>0, L_{r}=0, L_{p} \geq 0, K_{z}>0, K_{x}=0$ & Semiparametric VAR \\
$L_{u} \geq 0, L_{r}>0, L_{p} \geq 0, K_{z}=0, K_{x}=0$ & Gaussian ARCH \\
$L_{u} \geq 0, L_{r}>0, L_{p} \geq 0, K_{z}>0, K_{x}=0$ & Semiparametric ARCH \\
$L_{u} \geq 0, L_{r} \geq 0, L_{p}>0, K_{z}>0, K_{x}>0$ & Nonlinear Nonparametric \\
\hline \hline
\end{tabular}



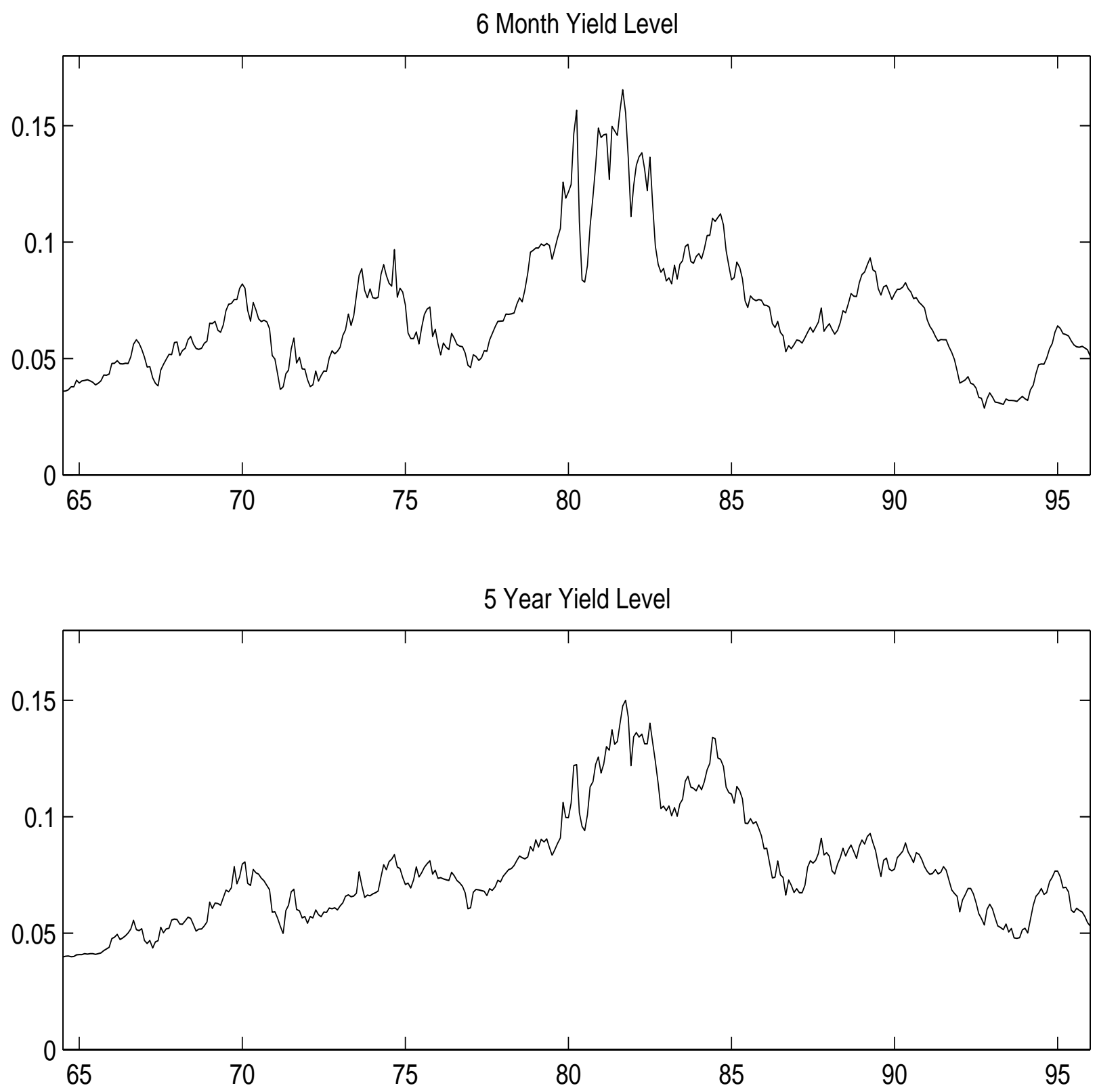

Figure 1: Observed Short Rate and Long Rate. 


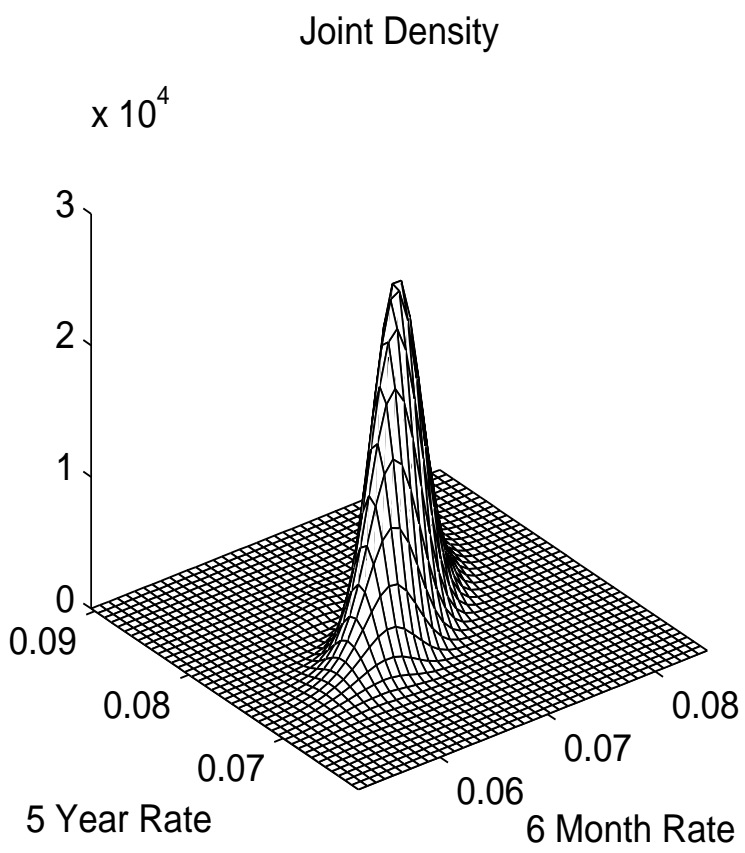

Contour at $10 \%$

$\times 10^{4}$
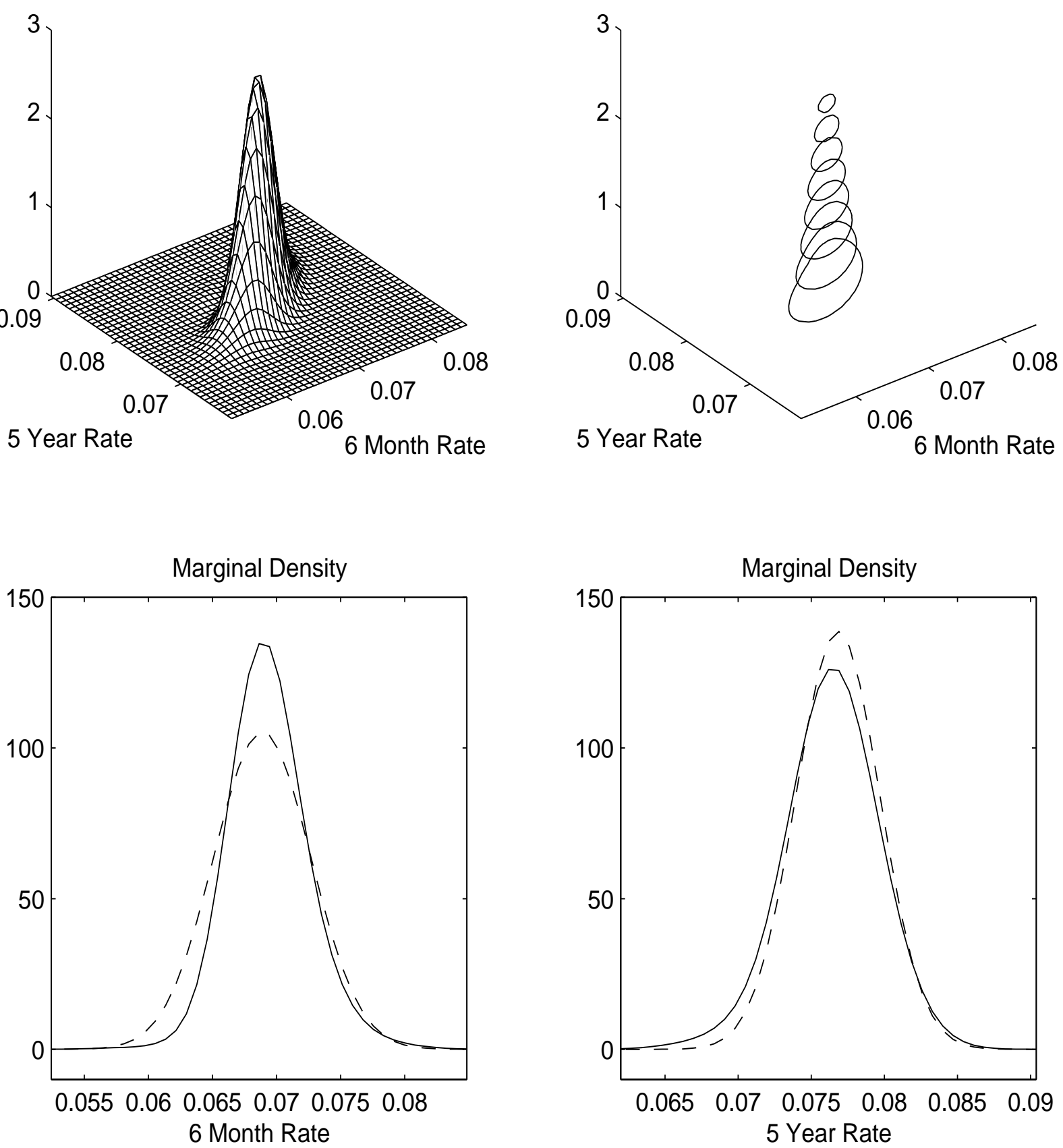

Figure 2: SNP Joint and Marginal Densities. The top-left panel is the joint SNP density, and the top-right is the quantile contour plot at $10 \%$ intervals. For bottom panels, the solid lines "___" are SNP marginal densities and the dashed lines "_ _ _" are normal densities with the same mean and variance. 

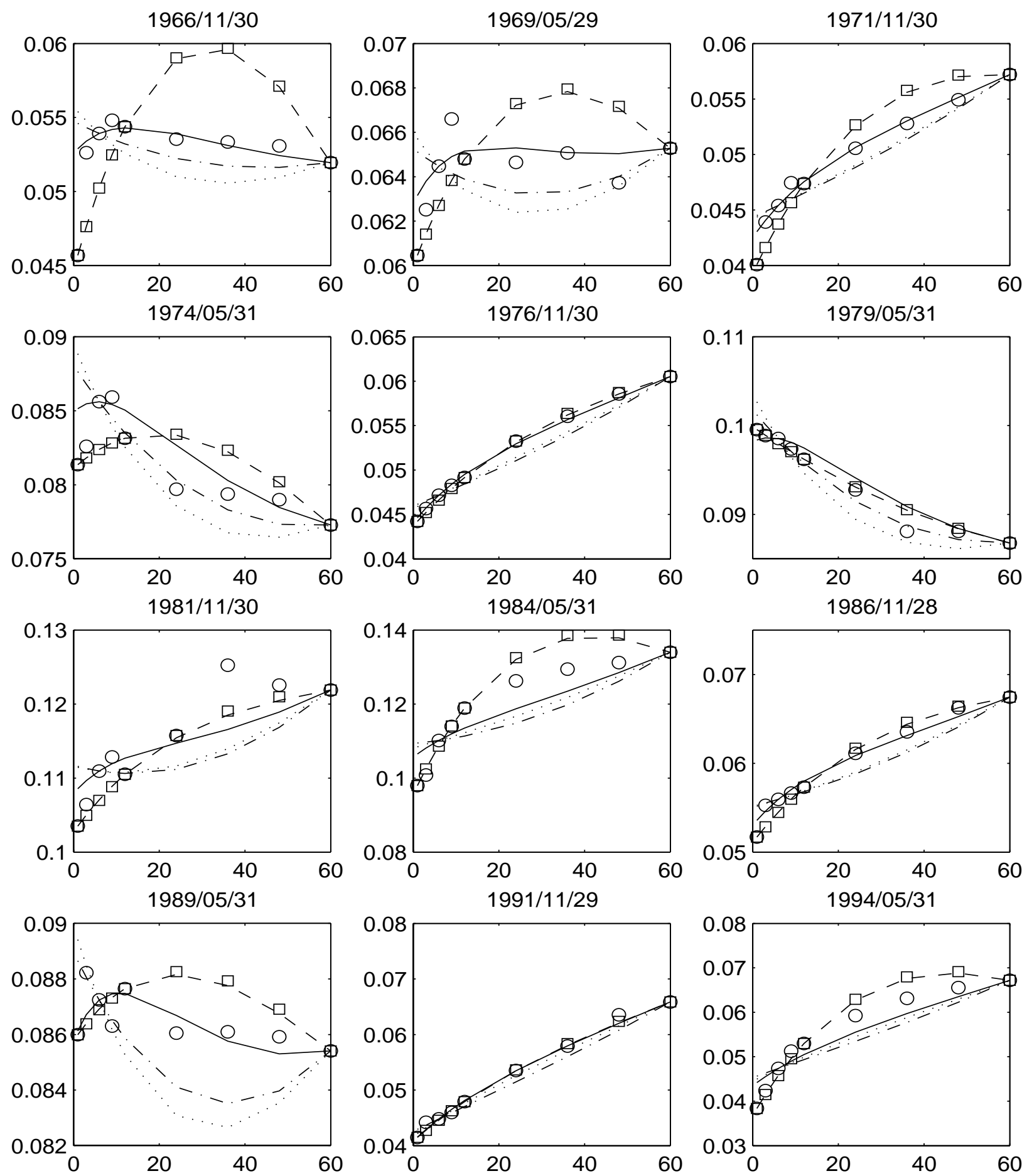

Figure 3: Point in Time Yield Curve. The symbols are respectively, "o o o" observed yield, "...." 2-Factor[CIR], "....." 2-Factor[RSc], "—-" 2-Factor[RS], "- - -" 3-Factor[CIR], " $\square$ $\square$ "3-Factor[AF]. 

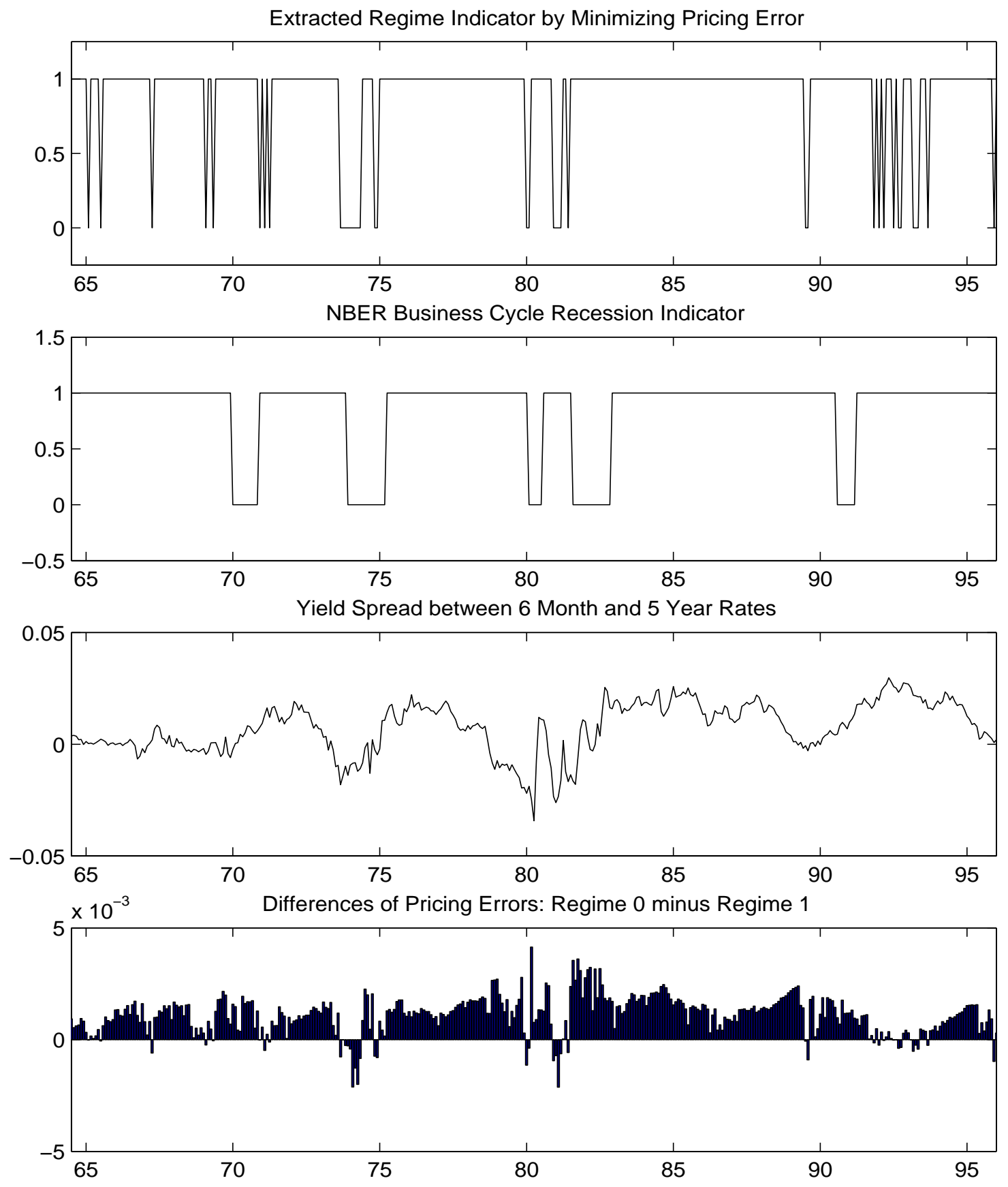

Figure 4: Regime Classifications and Economic Indicators in 2-Factor[RS] Model. 

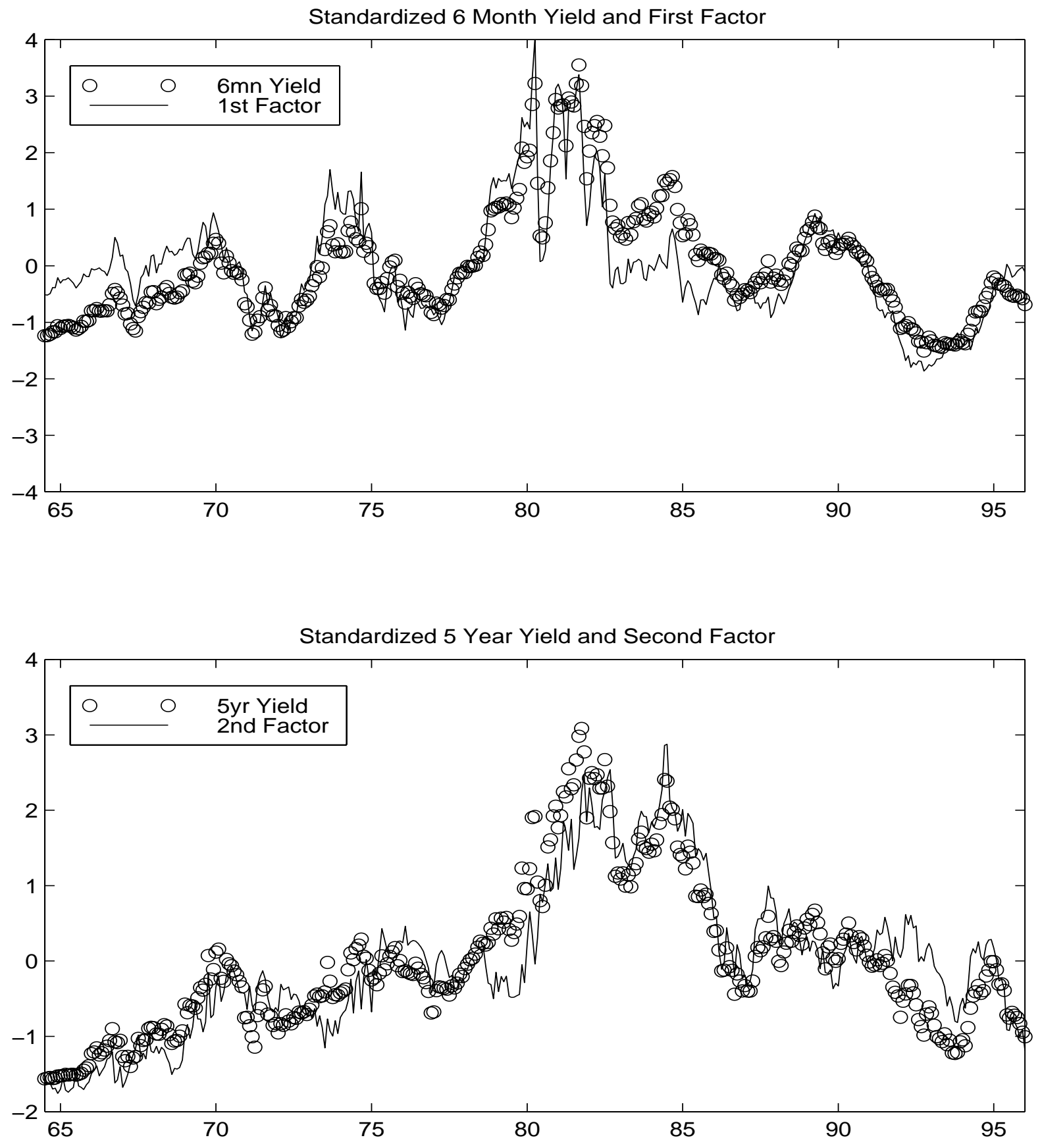

Figure 5: Square-Root Factors and Observed Yields in 2-Factor[RS] Model. 

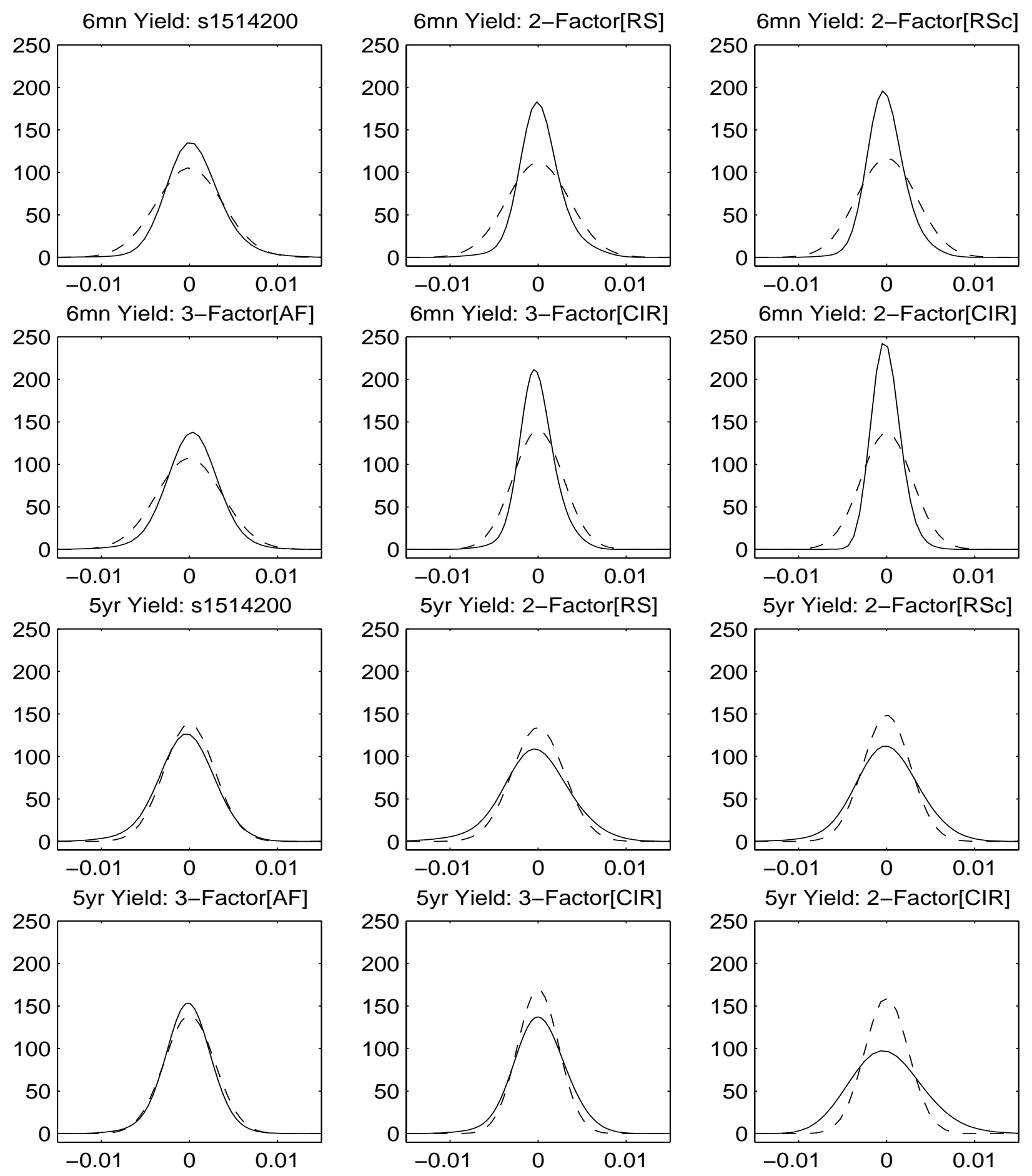

Figure 6: Projected and Reprojected Densities. The symbols are: "- - _" Gaussian densities with same mean and variance, "__ " projected or reprojected densities. 

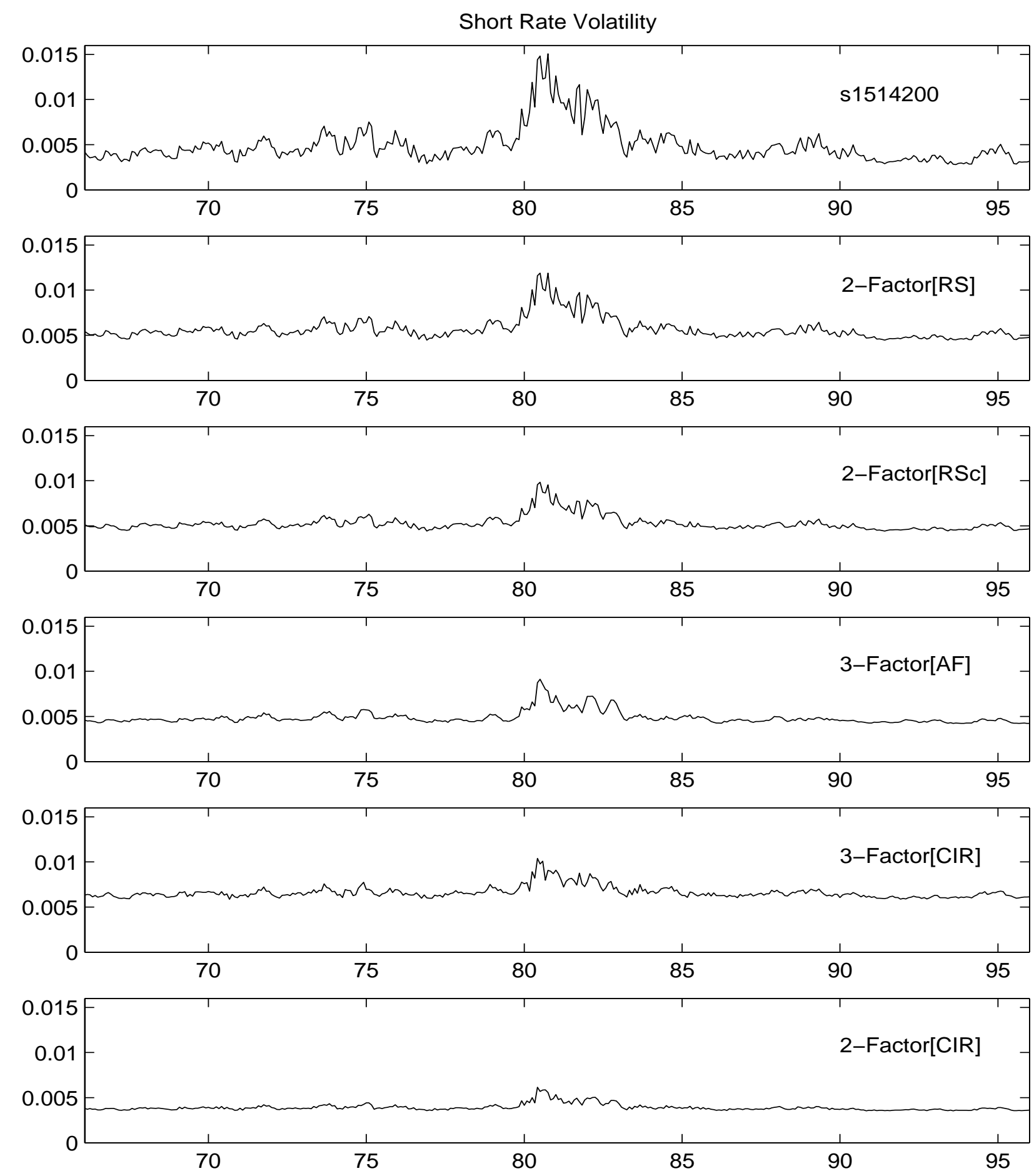

Figure 7: Projected and Reprojected Conditional Standard Deviation of 6 Month Yield. 


\section{Long Rate Volatility}
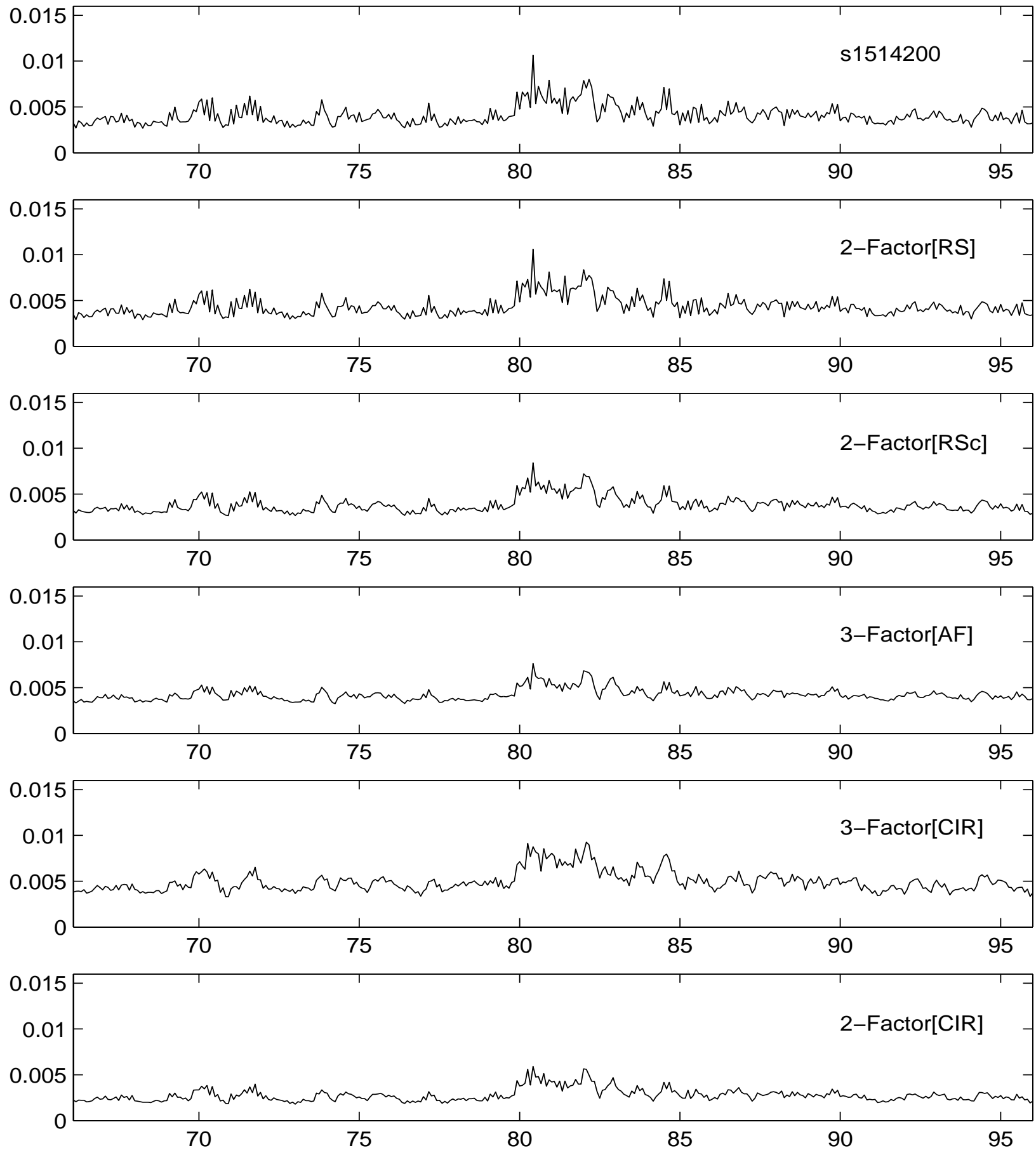

Figure 8: Projected and Reprojected Conditional Standard Deviation of 5 Year Yield. 

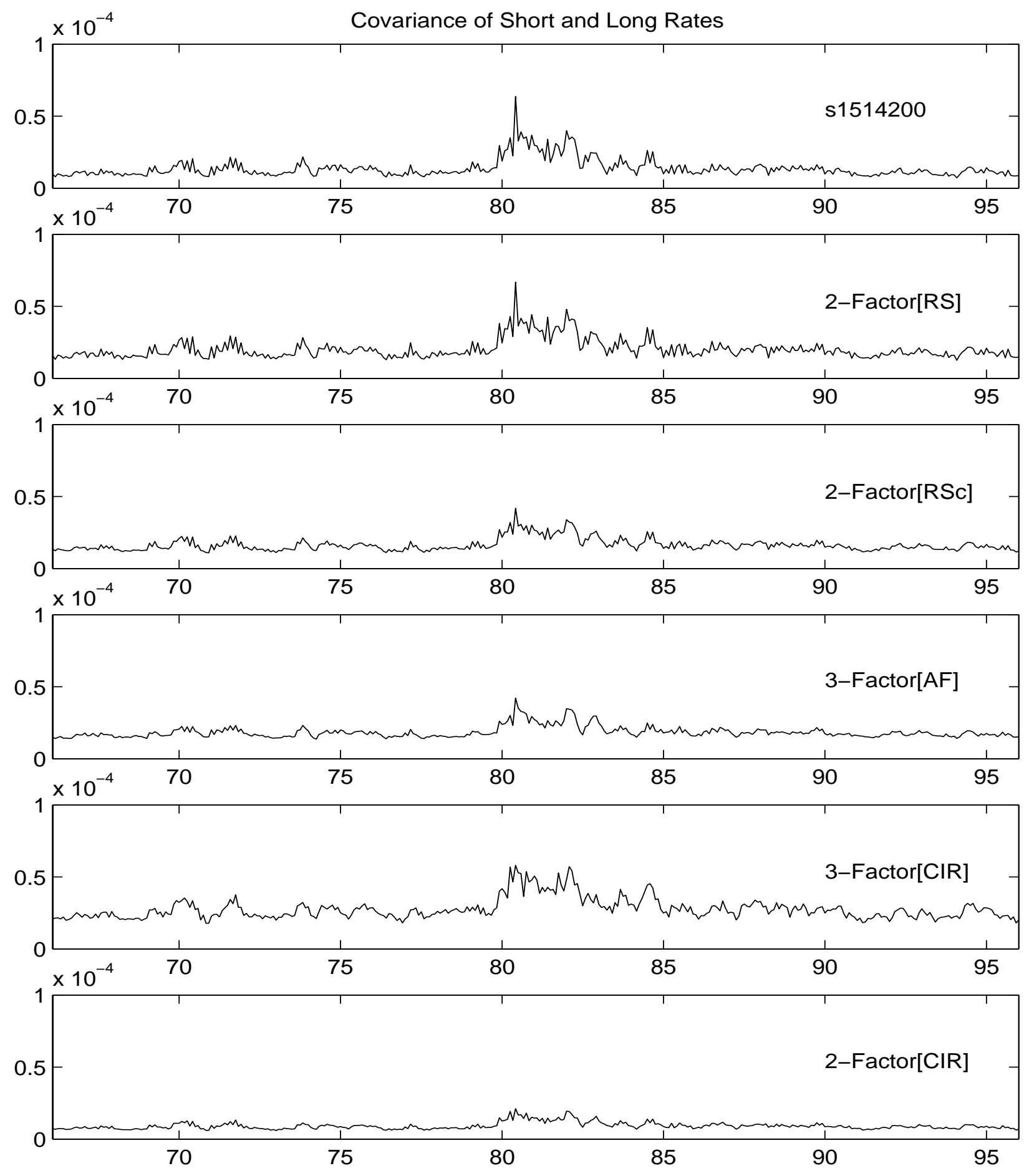

Figure 9: Projected and Reprojected Conditional Covariance of 6 Month and 5 Year Yields. 


\section{Correlation of Short and Long Rates}
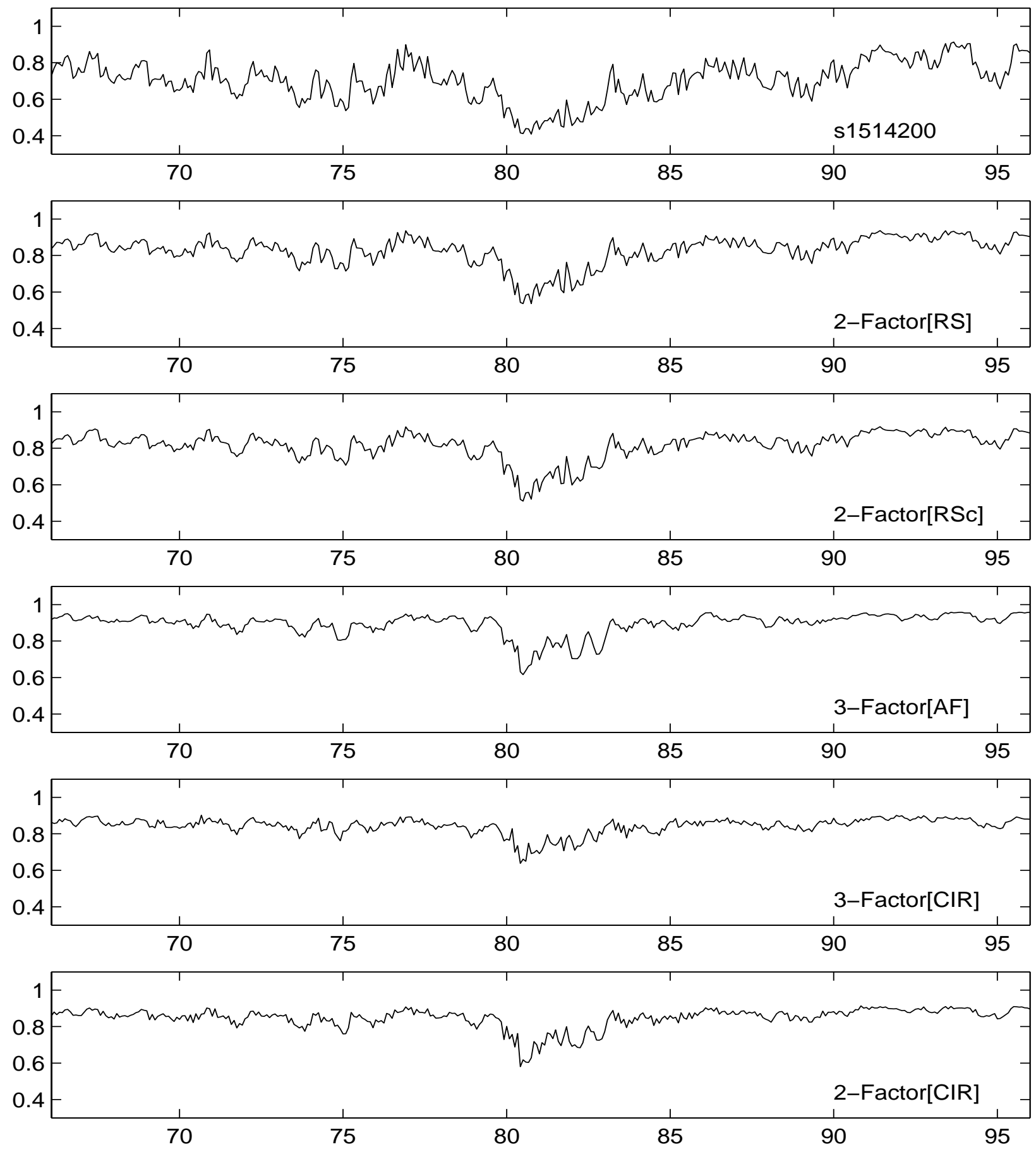

Figure 10: Projected and Reprojected Conditional Correlation of 6 Month and 5 Year Yields. 